\title{
PETROLOGICAL AND GEOCHEMICAL INVESTIGATIONS ON DOKHAN VOLCANICS AT WADI UM SIDRA-WADI UM ASMER AREA, NORTH EASTERN DESERT, EGYPT
}

\author{
Seddiek, S. H. ${ }^{1}$, El Afandy, A. H. ${ }^{1}$, El Kaliouby, B. A. ${ }^{2}$, Eliwa, H. A. ${ }^{3}$ and Khamis, H. A. ${ }^{1}$ \\ 1- Nuclear Materials Authority, P.O. 530, El-Maadi, Cairo, Egypt. \\ 2- Geology Department, Faculty of Science, Ain Shams University, \\ 3- Geology Department, Faculty of Science, Minufiya University, Egypt
}

\begin{abstract}
The present work provides the geology, geochemistry, and petrogenesis of the Dokhan volcanics rocks exposed at the areas surrounding Wadi Um Sidra and Wadi Um Asmer in the northern Eastern Desert of Egypt, between Latitudes $27^{\circ} 14^{\prime}$ and $27^{\circ} 26^{\prime} \mathrm{N}$ and Longitudes $33^{\circ} 8^{\prime}$ and $33^{\circ} 22^{\prime}$ E. The Dokhan volcanic rocks are exposed in the central (Gabal Dokhan) and the eastern parts of the mapped area. They cut by several sets of faults causing distinctive rugged topography, whereas narrow wadis run through these rocks. Based on the field and petrographical studies, the Dokhan volcanics are represented by a thick sequence of stratified lava flows of andesites, dacites, rhyolites and scarce amounts of basaltic rocks with pyroclastics which are composed of tuffs, agglomerates and ignimbrite. The Dokhan volcanics are intruded by monzogranites, syenogranites and alkali feldspar granites with sharp and irregular contacts, and sometimes are represented by large apophyses and several offshoots in syenogranites and as roof pendant over the monzogranites. Geochemically, these rocks have shown metaluminous to slightly peraluminous, medium to high- $\mathrm{K}$ calc- alkaline affinities and are unmetamorphosed with dominantly porphyritic texture and they enriched in LREE. The REE patterns of the rhyolites are characterized by wing shape with strong negative $\mathrm{Eu}$ anomaly, reflecting plagioclase fractionation.

The studied Dokhan volcanic rocks have geochemical characteristics similar to the rocks developed in volcanic arc setting in subduction environment rather than transitional or post-collisional setting. Rhyolites have geochemical characteristics of lavas erupted in collisional setting.The systematic variations of major oxides and trace element abundances along continuous trends of decreasing $\mathrm{Al}_{2} \mathrm{O}_{3}$, $\mathrm{CaO}, \mathrm{MgO}, \mathrm{Fe}_{2} \mathrm{O}_{3}, \mathrm{TiO}_{2}, \mathrm{P}_{2} \mathrm{O}_{5}, \mathrm{Ni}, \mathrm{Cr}$ and $\mathrm{Sr}$ and increasing $\mathrm{K}_{2} \mathrm{O}, \mathrm{Rb}, \mathrm{Nb}$ and $\mathrm{Zr}$ with increasing $\mathrm{SiO}_{2}$ can be interpreted in terms of fractional crystallization. The similarity and parallel nature of the normalized REE patterns with increasing total REE contents and decreasing Eu/Eu* with increasing $\mathrm{SiO}_{2}$ imply a major role of crystal fractionation during the evolution of these rocks.
\end{abstract}

Keywords: Dokhan volcanics; Um Sidra; Um Asmer; Calc-alkaline; Eastern Desert.

\section{INTRODUCTION}

The basement complex of Egypt in the Eastern Desert and south Sinai represents the northwestern segment of the Arabian-Nubian Shield (ANS), which represents the largest Neoproterozoic juvenile continental crust on Earth ( $\sim 3$ million km² ${ }^{2}$, Stern, 2002; Gradstein, et al., 2004; Be'eri-Shlevin et al., 2009, 2012; Fritz et al., 2013). It comprises dismembered ophiolites, island-arc related volcano-sedimentary successions, gabbroic and granitoids intrusions. The ANS was evolved during the Pan-African tectonothermal orogeny spanning the time interval $\sim 900$ to $550 \mathrm{Ma}$ (Kröner et al.1992).

The converging processes (e.g. subduction, accretion of intra-oceanic island arcs, back arc basins, and micro-continental plates, followed by crustal thickening) play an essential role in the evolution of the Egyptian basement complex during time between about 900 and 614 Ma (Stern and Hedge 1985). The terminal stage (614-550 Ma) of crustal evolution is characterized by the eruption of the Dokhanvolcanics (Stern and Hedge, 1985; Kröner et al., 1992; Stern, 1994), deposition of molasse-type Hammamat 
Seddiek, et al.

sediments (Grothaus et al. 1979; Akaad and Noweir 1980), and shallow emplacement of the younger granites (El Gaby, 1975).

The term "Dokhan volcanics" refers to varicolored thick sequence of lava flows and their pyroclastics of predominantly andesitic to rhyolitic composition in association with ignimbritic rhyolites (Basta et al. 1980; Stern and Gottfried 1986; Abdel Rahman 1996). The Dokhan Volcanics are obviously abundant in the northern Eastern Desert (NED) and southern Sinai with few scattered occurrences in the central and southern Eastern Desert. They are representing the second major volcanic episode recognized in the Precambrian crust of the Arabian-Nubian Shield. The Dokhan volcanics differ from the younger metavolcanics in their greater abundance of acidic volcanics (dacite and rhyolite), higher potassic nature, and common presence of ignimbrites and welded tuffs (El Gaby et al. 1991).

Several studies have been carried out on the Dokhan Volcanics (E.g.: Basta et al., 1980; El-Gaby et al., 1989; Abdel-Rahman, 1996; Mohamed et al., 2000; Moghazi, 2003; El-Sayed et al., 2004; Eliwa et al., 2006, 2010, 2014; Breitkreuz et al., 2010; El-Bialy, 2010;Azer and Farahat, 2011; Khalaf et al., 2011; Khalaf, 2012 \& 2013). These show that, these volcanics have medium- to high-K calc-alkaline affinities and are unmetamorphosed with dominantly porphyritic texture and felsic to intermediate composition that have been formed in subaerial environment.

Based on SHRIMP U-Pb zircon ages of Dokhan Volcanics from eight major occurrences in the NED (Breitkreuz et al., 2010) have recently indicated that the Dokhan Volcanics were erupted through two main pulses of volcanic activity: 630-623 Ma and 618-592 Ma. Their determined ages along with the previously published results on the radiogenic ages for Dokhan Volcanics from the Eastern Desert (e.g. Stern and Hedge, 1985; Abdel-Rahman and Doig, 1987; Wilde and Youssef, 2000) and those from Sinai (Be'eri-Shlevin et al., 2011) place the eruption of the Dokhan Volcanics within the 590-630 Ma time span.

Fractional crystallization of basaltic magma coupled with slight crustal contamination is generally the preferred processes for their magma genesis and controlled the magmatic evolution (e.g.: El Gaby et al., 1989; Abdel-Rahman, 1996; Mohamed et al., 2000; Moghazi, 2003; El-Sayed et al., 2004; Eliwa et al., 2006, 2014; El-Bialy, 2010; Abdel Wahed et al., 2012; Khalaf, 2012). However, interpretation of tectonic setting are still controversial, especially whether they have been formed (1)in a subduction environment (Hassan and Hashad, 1990; Abdel-Rahman, 1996; Hassan et al., 2001;Abdel Wahed et al., 2012), (2) in association with extension after crustal thickening (Stern et al., 1984; Stern and Gottfried, 1986; Mohamed et al., 2000), or (3) during transition between subduction and extension (Ressetar and Monard, 1983; Moghazi, 2003; El-Sayed et al., 2004; Eliwa et al., 2006, 2014; El-Bialy, 2010; Azer and Farahat, 2011; Be'eri-Shlevin et al., 2011; Khalaf, 2012).

The present work concerns mainly with the geology, petrography, geochemistry, and petrogenesis of the Dokhan volcanics rocks exposed at the areas surrounding Wadi Um Sidra and Wadi Um Asmer in the northern Eastern Desert of Egypt.

\section{GEOLOGICAL SETTING AND FIELD OBSERVATIONS}

The investigated area is located at about $50 \mathrm{~km}$ northwest of Hurghada city in the North Eastern Desert (NED) of Egypt. A new geological map was produced for the area, which delineated by latitudes $27^{\circ} 14^{\prime}$ and $27^{\circ} 26^{\prime} \mathrm{N}$ and longitudes $33^{\circ} 8^{\prime} \mathrm{E}$ (Fig. 1). Chronologically, the basement rock units of the area from the oldest to the youngest are metavolcanics, Dokhan volcanics, monzogranites, syanogranites, and alkali feldspar granites. All of these rock unites are dissected by numerous dykes of variable compositions trending mainly NE-SW, NNE-SSW and NNW-SSE. The Dokhan volcanics covers a vast area of the mapped area including the type locality Gebel Dokhan (1705 $\mathrm{m}$ a.s.l), which is characterized by rugged topography and crenulated peaks as well as gentle to deep slopes.

The Dokhan volcanics in the area are conspicoudely fractured with narrow spacing joints. They exhibit a specific layered appearance, implying successive eruptions. They are intruded by syenogranites through sharp and irregular intrusive contacts in the form of large apophyses and several offshoots (Fig. 2a). In 


\section{Petrological and geochemical investigations on Dokhan Volcanics}

places, the Dokhan volcanics occur as roofpendant over the monzogranites (Fig. 2.b), where they were silicified and in some parts brecciated, especially along and directly near the contacts. They are represented by a thick sequence of stratified lava flows of intermediate to acidic composition, together with a few intercalations of pyroclastics (tuffs and volcanic breccias). The lava flows occur as sheets, generally characterized by columnar jointing with amygdaloidal and fluidal structures. They are cut by more than one set of faults causing distinctive rugged topography, whereas several narrow wadis are running through these rocks. The Dokhan volcanics are hard and massive with variable colors ranging from pink, brownish red and red in acidic varieties, while the intermediate ones are grey to dark grey or green when chloritized.

Fig.1: Geological map of W. Um Sidra and W. Um Asmer area, NED.

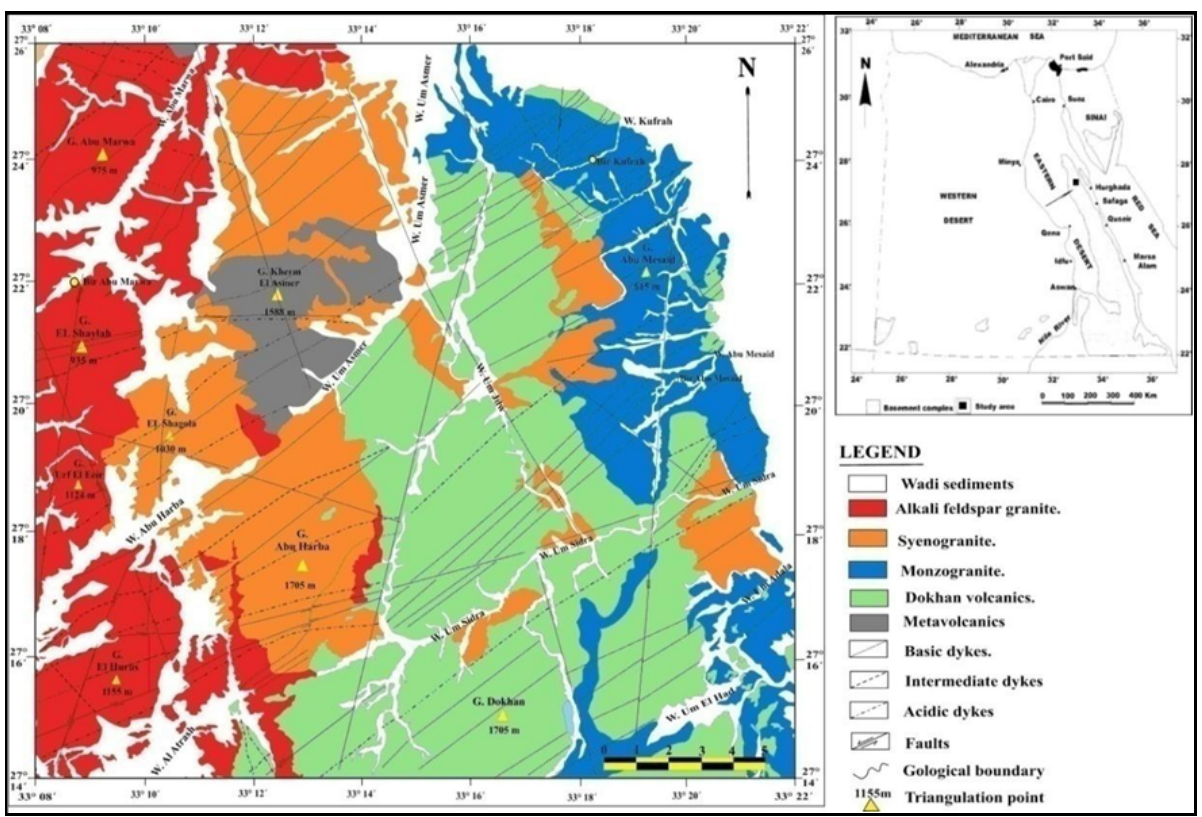

The andesites, dacites, and rhyolites are the most abundant rocks of the Dokhan volcanics. Basaltic rocks are scarce, and in many cases are found only at the base of the succession. Lavas are commonly porphyritic. The main alteration processes affected these rocks are chloritization, sericitization, and silicification. The pyroclastics occur as lenticular intercalations between the lava flows and represented by tuffs, volcanic breccia and agglomerates. A few sheets or small lenticular masses of very fine-grained violet ignimbrite are also encountered especially along the eastern periphery of G. Dokhan.

A great number of dykes cut through the Dokhan volcanics. They are arranged in subparallel relatively high ridges and generally following the NE-SW, ENE-WSW and E-W directions. Quartz veins and veinlets cut the Dokhan volcanics especially at their contacts with the syenogranites. They predominate in the NE-SW, NNE-SSW and N-S trends with a steep angle dip.

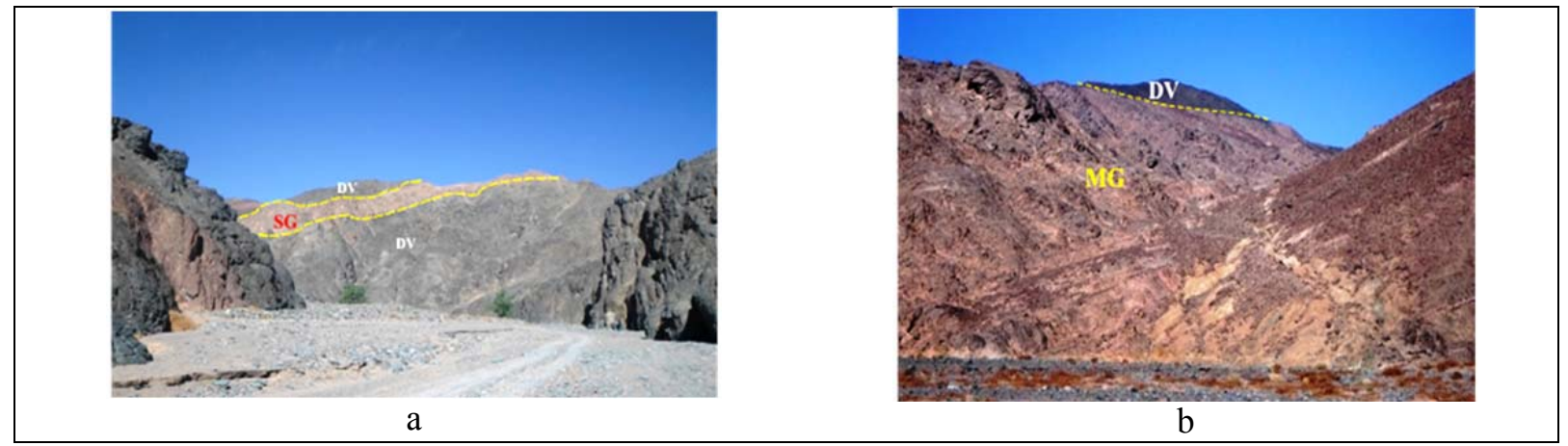

Fig. 2: a) Offshoots of syenogranites (SG) into the Dokhan volcanic (DV), Um Sidra; looking W. b): Roof pendant of the Dokhan volcanics (DV) over the monzogranites (MG), G. Abu Mesaid. 
Seddiek, et al.

\section{PETROGRAPHY}

The Dokhan Volcanics are mainly represented by basaltic andesites, andesites, dacites and rhyolites. These volcanics are generally porphyritic with phenocrysts constituting about $20-60 \%$ of the total rock volume:

Basaltic andesites consist of plagioclases together with amphibole and augite set in a fine-grained groundmass. Plagioclases are ubiquitous as anhedral to subhedral tabular to prismatic (up to $0.4 \mathrm{~cm}$ ) phenocrysts of labradorite to andesine (An40-60), partially altered to suassurite, chlorite, and epidote (Fig 3a). Augite phenocrysts are equant, simply twinned, and variably altered to actinolite and chlorite around their margins. Hornblende forms subhedral prismatic phenocrysts include minute prismatic crystals of apatite and is occasionally altered to chlorite around their margins. The common accessories are opaques and apatite, while suassurite, epidote and chlorite are secondary minerals.

Andesites display glomeroporphyritic texture and flow structure defined by the parallel alignment of tabular plagioclase phenocrysts. Andesites are composed of plagioclase and hornblende phenocrysts embedded in fine-grained groundmass. Euhedral to subhedral, tabular to prismatic phenocrysts of andesine (An35-45) are generally arranged in subparallel orientation, suggesting flow texture. Some of the plagioclase phenocrysts are partially altered to sericite and epidote, especially along cores and fractures. Hornblende phenocrysts form subhedral to euhedral prismatic to sphenoid shape. Some hornblende phenocrysts poikilitically contain inclusions of apatite, quartz, and opaques. Quartz occurs as small crystals in association with plagioclase microlites in the groundmass; sometimes they intergrown forming myrmekitic texture (Fig 3b). Biotite is generally a groundmass phase. The main accessory minerals are apatite and opaques, while chlorite, epidote and clay minerals are secondary minerals.

The basaltic andesites as well as andesites have suffered from low-grade regional metamorphism of greenschist facies as indicated from the replacement of both hornblende and augite by chlorite, epidote and iron oxides.

Dacites consist of oligoclase (An20-30), quartz, biotite and potash feldspars as well as few hornblende phenocrysts embedded in a fine-grained groundmass, which is formed of small lathes of plagioclase, quartz, biotite, epidote and chlorite. Hornblende form seuhedral to subhedral, greenish brown prismatic crystals or as clusters of fibrous or columnar aggregates. It is show by rims of $\mathrm{Fe}-\mathrm{Ti}$ oxides and chlorite. Quartz occurs as fine grains and common in the groundmass rather than phenocrysts. Some crystals show undulose extinction. Biotite is present in the form of subhedral to anhedral flakes of light brown color (Fig 3c). Most of biotite flakes exhibit slight alteration to chlorite especially along peripheries and cleavage planes. Apatite, zircon, and sphene are common accessories. Kaoline, sericite, chlorite, and epidote are secondary minerals.

Rhyolites have phenocrysts of K-feldspar, quartz and some biotite embedded in a fine-grained groundmass. Spherulites are common and are composed of radiating and fibrous aggregates of alkali feldspar intergrowths (Fig 3d). Alkali feldspar occurs as subhedral prismatic to tabular Carlsbad twinned sanidine phenocrysts up to $2.8 \mathrm{~mm}$ in length and $1.2 \mathrm{~mm}$ in width or as minute crystals spread throughout the groundmass. It is partially altered to kaoline. Quartz forms subhedral to anhedral elongated $(1.5 \mathrm{x} 1$ $\mathrm{mm}$ size) and equant phenocrysts. Plagioclases (An14-20) occur as subhedral to euhedral prismatic and tabular phenocrysts. Biotite flakes are partially replaced by chlorite. Accessories are opaques, apatite, sphene and zircon, whereas the secondary minerals are sericite, chlorite and kaoline.

Pyroclastics are interlayers within the volcanic flows and are represented by tuffs, lapilli tuffs, crystal tuffs and ignimbrites. The lithic crystal lapilli tuffs are composed of lithic and crystal fragments embedded in very fine-grained tuffaceous matrix (Fig 3e). The lithic fragments are andesitic, dacitic and rhyolitic in composition. The andesitic fragments are porphyritic and composed of plagioclases and chloritized hornblende embedded in a fine-grained groundmass. The dacitic fragments are composed of plagioclase micro-phenocrysts set in a fine-grained groundmass of plagioclase laths and quartz. The rhyolitic 
fragments are composed of microcrystalline aggregates of $\mathrm{K}$ - feldspar and quartz. The crystal fragments are represented by plagioclases and quartz crystals.

The crystal lapilli tuffs are composed of crystal fragments embedded in a fine-grained matrix of plagioclases and quartz. The crystal fragments are represented by plagioclases, quartz and opaques of variable sizes. Plagioclases occur as anhedral crystals altered to suassurite and epidote. Opaques occur as small irregular grains dispersed in the matrix.

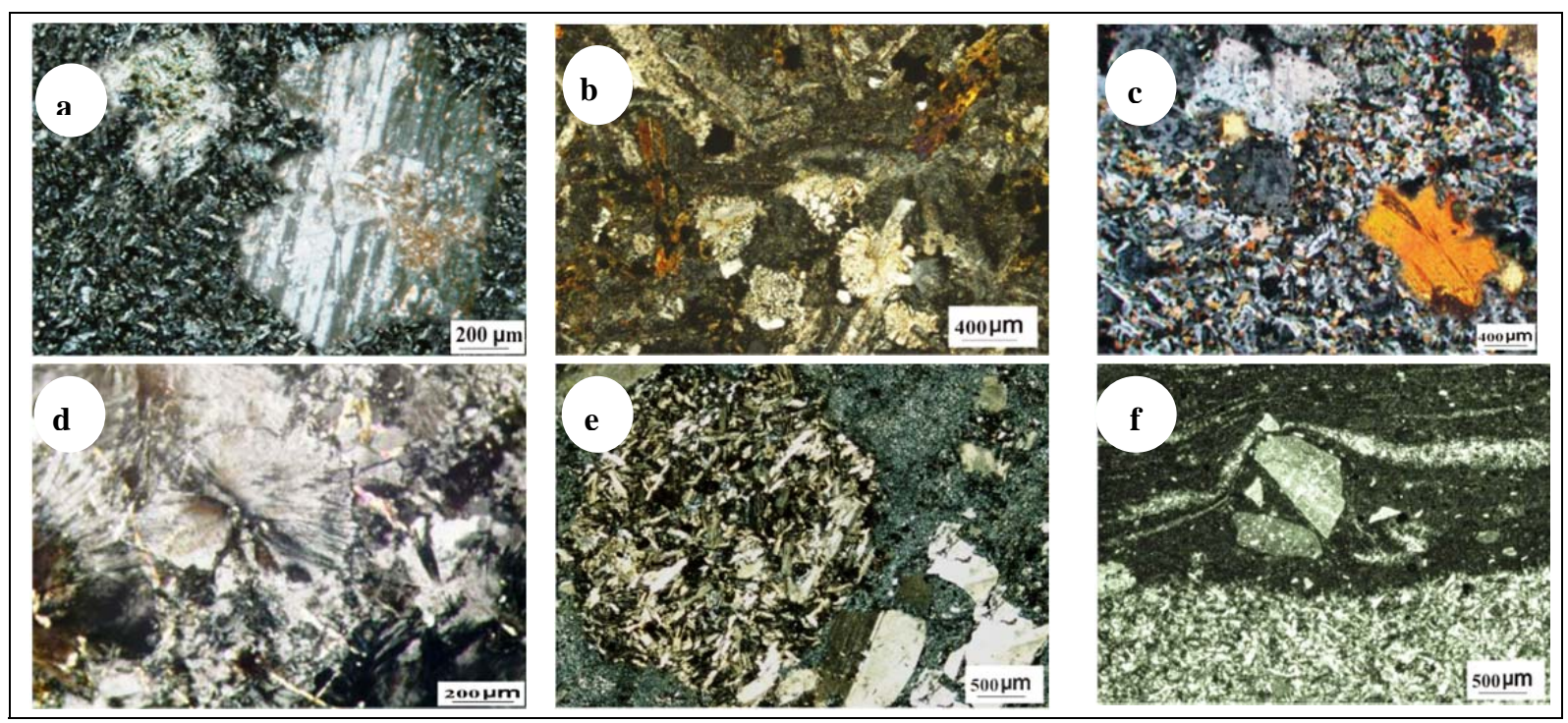

Fig. 3: Photomicrographs of Dokhan volcanics: a) Saussuritized plagioclase phenocrysts and epidote alteration, basaltic andesite. C.N, (b) Quartz intergrown with plagioclase forming myrmekitic intergrowths in andesites, C.N, (c) Irregular flakes of biotite embedded in fine-grained groundmass in dacite, C.N, (d) Spheriulitic texture composed of cryptocrystalline quartz and feldspars in rhyolites, C.N, (e) Andesite fragments and plagioclase laths in the lithic crystal lapilli tuff, C.N, (f) Curving of fiamme around the crystal fragments in ignimbrites, C.N.

Welded crystals tuffs (ignimbrites) are characterized by flow laminations. They contain subangular to subrounded lithic and crystal fragments set in glassy groundmass. The laminae have a lenticular form and pinch out against each other forming fiamme texture characteristic of ignimbrites. The fiamme are, elongated or lenticular in shape and have flame-like ends with variable width/length ratios. The alignment of the fiamme is attributed to welding compaction or diagenetic compaction (McPhie et al., 1993). The fiamme are commonly sinuously curved especially around the fragments in the glass matrix (Fig 3f), reflecting the effect of compaction. The rock fragments are mostly andesitic. The crystal fragments are dominantly plagioclase. The plagioclase crystals are subhedral, twinned and variably sericitized. Some of the plagioclase crystals are oriented with their long axes parallel to the glass matrix flow. The plagioclase fragments are slightly altered to epidote or sericite, or variably replaced by carbonates. The plagioclase fragments are commonly embayed and extensively corroded by the glassy matrix. The glassy matrix show well-developed welding around the crystal and the lithic fragments and wrapping around them.

\section{GEOCHEMISTRY}

Thirty-five samples of the studied Dokhan volcanics were chemically analyzed for major oxides and trace elements using Rigaku RIX 2000 X-ray fluorescence (XRF) spectrometer and fifteen samples were analyzed for rare earth elements (REEs) using inductively coupled plasma- mass spectrometry (ICP-MS). All analyses were carried out at the Department of Earth Sciences, Faculty of Science, Naruto University, Japan. The results of the major oxides and trace elements including CIPW norms and some calculated parameters are listed in Table 1, while the REEs are listed in Table 2.

\section{Geochemical classification}

On the total alkalis vs. silica binary diagram of Le Bas et al., (1986) (Fig. 4), the Dokhan volcanics fall in calc-alkaline basaltic andesites, andesites, dacite, and rhyolites. 
Seddiek, et al.

Table 1: Major oxides, trace and rare earth elements, as well as, CIPW norm with some parameters and geochemical ratios for the studied Dokhan Volcanics.

\begin{tabular}{|c|c|c|c|c|c|c|c|c|c|c|c|c|c|c|c|c|c|c|c|}
\hline Rock type & Basalti & ic andesite & & & & & & Andesit & & & & & & & & & & & Dacite \\
\hline Sample No. & GDP-1 & GDP-2 & GDP-5 & GDP-9 & GDP-13 & GDP-15 & Average & GD -1 & GD-4 & GD-9 & GD-10 & GD-11 & GD-19 & GDP- 3 & GDP- 8 & GDP- 12 & GDP -14 & Average & GD-3 \\
\hline $\mathrm{SiO}_{2}$ & 55.71 & 53.89 & 54.96 & 55.33 & 56.65 & 56.10 & 55.44 & 60.28 & 60.78 & 60.08 & 59.49 & 61.33 & 61.48 & 58.34 & 57.86 & 60.10 & 57.75 & 59.75 & 64.25 \\
\hline $\mathrm{TiO}_{2}$ & 1.34 & 1.43 & 1.28 & 1.30 & 1.31 & 1.14 & 1.30 & 0.98 & 0.89 & 0.92 & 1.02 & 0.89 & 0.86 & 1.13 & 1.32 & 1.12 & 1.45 & 1.06 & 0.90 \\
\hline $\mathrm{Al}_{2} \mathrm{O}_{3}$ & 15.10 & 17.95 & 16.13 & 15.18 & 15.92 & 16.15 & 16.07 & 15.81 & 15.02 & 16.90 & 15.47 & 14.78 & 14.82 & 16.92 & 17.36 & 17.45 & 18.38 & 16.29 & 15.79 \\
\hline $\mathrm{Fe}_{2} \mathrm{O}_{3}$ & 8.40 & 8.27 & 8.12 & 9.40 & 7.92 & 7.96 & 8.35 & 5.92 & 7.83 & 6.11 & 7.73 & 6.43 & 6.85 & 7.92 & 7.15 & 8.35 & 7.85 & 7.21 & 4.85 \\
\hline MnO & 0.12 & 0.32 & 0.13 & 0.12 & 0.10 & 0.11 & 0.15 & 0.70 & 0.08 & 0.10 & 0.11 & 0.11 & 0.09 & 0.10 & 0.14 & 0.11 & 0.16 & 0.17 & 0.10 \\
\hline MgO & 5.10 & 3.50 & 4.97 & 4.90 & 3.74 & 4.92 & 4.52 & 2.87 & 2.59 & 2.27 & 3.00 & 2.81 & 2.56 & 2.68 & 2.96 & 2.12 & 3.12 & 2.70 & 1.46 \\
\hline $\mathrm{CaO}$ & 6.54 & 6.74 & 7.28 & 6.45 & 6.10 & 6.64 & 6.63 & 4.15 & 4.06 & 4.49 & 4.87 & 4.48 & 3.96 & 5.74 & 5.17 & 4.25 & 4.23 & 4.54 & 2.45 \\
\hline $\mathrm{Na}_{2} \mathrm{O}$ & 3.78 & 3.72 & 4.05 & 3.94 & 4.07 & 3.34 & 3.82 & 4.07 & 4.68 & 4.53 & 4.12 & 4.42 & 4.18 & 4.18 & 4.25 & 3.89 & 4.09 & 4.24 & 5.56 \\
\hline $\mathbf{K}_{2} \mathbf{O}$ & 2.19 & 1.57 & 1.14 & 1.72 & 2.26 & 1.72 & 1.77 & 3.01 & 1.99 & 2.26 & 2.12 & 2.12 & 2.66 & 1.73 & 2.09 & 2.01 & 1.60 & 2.16 & 2.88 \\
\hline $\mathbf{P}_{2} \mathbf{O}_{5}$ & 0.43 & 0.38 & 0.41 & 0.35 & 0.43 & 0.38 & 0.40 & 0.28 & 0.27 & 0.39 & 0.36 & 0.26 & 0.25 & 0.34 & 0.49 & 0.24 & 0.31 & 0.32 & 0.32 \\
\hline L.O.I & 1.14 & 1.96 & 1.51 & 1.30 & 1.40 & 1.52 & 1.47 & 2.12 & 2.02 & 1.94 & 1.29 & 1.96 & 2.10 & 0.90 & 1.39 & 0.34 & 1.05 & 1.51 & 1.41 \\
\hline Total \% & 99.85 & 99.73 & 99.98 & 99.99 & 99.90 & 99.98 & 99.91 & 100.19 & 100.21 & 99.99 & 99.58 & 99.59 & 99.81 & 99.98 & 100.18 & 99.98 & 99.99 & 99.95 & 99.97 \\
\hline $\mathbf{Q}$ & 7.51 & 8.38 & 7.56 & 8.08 & 8.83 & 11.00 & 8.56 & 13.25 & 15.07 & 13.32 & 14.47 & 15.82 & 16.30 & 12.93 & 11.11 & 18.20 & 15.00 & 14.55 & 14.47 \\
\hline C & 0.00 & 0.00 & 0.00 & 0.00 & 0.00 & 0.00 & 0.00 & 0.00 & 0.00 & 0.00 & 0.00 & 0.00 & 0.00 & 0.00 & 0.00 & 1.72 & 2.97 & 0.47 & 0.00 \\
\hline Or & 12.94 & 9.28 & 6.74 & 10.16 & 13.36 & 10.16 & 10.44 & 17.91 & 11.76 & 13.36 & 12.53 & 12.53 & 15.72 & 10.22 & 12.35 & 11.88 & 9.46 & 12.77 & 17.02 \\
\hline Ab & 31.99 & 31.48 & 34.27 & 33.34 & 34.44 & 28.26 & 32.30 & 34.44 & 39.60 & 38.33 & 34.86 & 37.40 & 35.37 & 35.37 & 35.96 & 32.92 & 34.61 & 35.89 & 47.05 \\
\hline An & 17.77 & 27.64 & 22.47 & 18.65 & 18.49 & 23.99 & 21.50 & 15.92 & 14.10 & 19.10 & 17.46 & 14.23 & 13.82 & 22.30 & 22.12 & 19.52 & 18.96 & 17.75 & 9.62 \\
\hline Di & 5.97 & 0.00 & 5.47 & 5.45 & 3.73 & 2.28 & 3.82 & 1.69 & 1.16 & 0.00 & 0.96 & 2.83 & 1.21 & 0.32 & 0.00 & 0.00 & 0.00 & 0.82 & 0.00 \\
\hline Hy & 9.94 & 8.72 & 9.85 & 9.68 & 7.59 & 11.20 & 9.50 & 6.37 & 5.91 & 5.65 & 7.03 & 5.69 & 5.82 & 6.53 & 7.37 & 5.28 & 7.77 & 6.34 & 3.64 \\
\hline $\mathrm{Hm}$ & 0.26 & 0.68 & 0.28 & 0.26 & 0.21 & 0.24 & 0.32 & 1.50 & 0.17 & 0.21 & 0.24 & 0.24 & 0.19 & 0.21 & 0.30 & 0.24 & 0.34 & 0.36 & 0.21 \\
\hline Il & 8.40 & 8.27 & 8.12 & 9.40 & 7.92 & 7.96 & 8.35 & 5.92 & 7.83 & 6.11 & 7.73 & 6.43 & 6.85 & 7.92 & 7.15 & 8.35 & 7.85 & 7.21 & 4.85 \\
\hline Tn & 2.96 & 2.33 & 2.78 & 2.86 & 2.94 & 2.49 & 2.73 & 0.47 & 1.96 & 0.44 & 2.20 & 1.88 & 1.86 & 2.50 & 0.23 & 0.00 & 0.00 & 1.15 & 0.31 \\
\hline Ap & 1.02 & 0.90 & 0.97 & 0.83 & 1.02 & 0.90 & 0.94 & 0.66 & 0.64 & 0.92 & 0.85 & 0.62 & 0.59 & 0.81 & 1.16 & 0.57 & 0.73 & 0.76 & 0.76 \\
\hline Ba & 615 & 813 & 566 & 541 & 723 & 765 & 670 & 822 & 540 & 730 & 669 & 503 & 628 & 495 & 913 & 544 & 630 & 647 & 865 \\
\hline $\mathrm{Cr}$ & 195 & 126 & 142 & 270 & 143 & 110 & 164 & 178 & 132 & 102 & 116 & 144 & 113 & 30 & 71 & 47 & 48 & 98 & 103 \\
\hline $\mathbf{N i}$ & 75 & 51 & 73 & 101 & 67 & 66 & 72 & 69 & 33 & 21 & 48 & 38 & 32 & 20 & 10 & 32 & 16 & 32 & 23 \\
\hline $\mathbf{P b}$ & 6 & 6 & 8 & 6 & 10 & 12 & 8 & 8 & 7 & 9 & 8 & 8 & 8 & 14 & 15 & 5 & 10 & 9 & 13 \\
\hline $\mathrm{Ce}$ & 56 & 45 & 49 & 52 & 58 & 57 & 53 & 54 & 45 & 59 & 41 & 41 & 46 & 46 & 70 & 50 & 50 & 50 & 68 \\
\hline $\mathbf{R b}$ & 65 & 58 & 19 & 17 & 26 & 28 & 36 & 68 & 36 & 52 & 39 & 41 & 56 & 38 & 62 & 52 & 45 & 49 & 44 \\
\hline $\mathrm{Sr}$ & 702 & 732 & 1088 & 812 & 859 & 1035 & 871 & 605 & 843 & 1020 & 843 & 748 & 945 & 728 & 570 & 587 & 596 & 748 & 617 \\
\hline Nb & 11 & 7 & 6 & 7 & 10 & 6 & 8 & 8 & 7 & 8 & 6 & 7 & 7 & 10 & 12 & 15 & 13 & 9 & 9 \\
\hline $\mathbf{Y}$ & 21 & 21 & 15 & 16 & 27 & 15 & 19 & 14 & 18 & 16 & 17 & 18 & 19 & 28 & 29 & 28 & 32 & 22 & 21 \\
\hline $\mathrm{Zr}$ & 190 & 174 & 150 & 211 & 213 & 166 & 184 & 203 & 192 & 196 & 182 & 187 & 186 & 194 & 268 & 178 & 237 & 202 & 256 \\
\hline Th & 9.0 & 2.9 & 3.4 & 2.4 & 3.0 & 4.0 & 4.1 & 5.1 & 4.9 & 6.0 & 3.8 & 4.7 & 4.4 & 4.0 & 6.3 & 3.0 & 4.5 & 4.7 & 5.2 \\
\hline $\mathbf{U}$ & 1.1 & 1.0 & 0.9 & 0.8 & 1.1 & 1.0 & 1.0 & 1.9 & 1.8 & 1.9 & 1.1 & 1.8 & 1.7 & 1.1 & 1.9 & 1.0 & 1.3 & 1.5 & 1.7 \\
\hline $\mathbf{K} / \mathbf{R b}$ & 279.7 & 223.9 & 490.3 & 820.6 & 721.6 & 509.9 & 507.72 & 370.20 & 457.64 & 363.61 & 450.13 & 429.27 & 394.35 & 377.96 & 279.41 & 320.90 & 295.18 & 373.87 & 544.52 \\
\hline $\mathbf{R b} / \mathbf{S r}$ & 0.09 & 0.08 & 0.02 & 0.02 & 0.03 & 0.03 & 0.05 & 0.11 & 0.04 & 0.05 & 0.05 & 0.05 & 0.06 & 0.05 & 0.11 & 0.09 & 0.08 & 0.07 & 0.07 \\
\hline $\mathbf{R b} / \mathbf{Z r}$ & 0.34 & 0.33 & 0.13 & 0.08 & 0.12 & 0.17 & 0.20 & 0.33 & 0.19 & 0.26 & 0.21 & 0.22 & 0.30 & 0.20 & 0.23 & 0.29 & 0.19 & 0.24 & 0.17 \\
\hline $\mathrm{Sr} / \mathbf{Y}$ & 34.24 & 34.86 & 74.01 & 49.51 & 31.81 & 69.00 & 48.91 & 43.21 & 46.07 & 63.75 & 48.73 & 41.99 & 49.71 & 26.00 & 19.45 & 20.96 & 18.63 & 37.85 & 29.10 \\
\hline Y/Nb & 1.86 & 2.96 & 2.61 & 2.31 & 2.79 & 2.50 & 2.51 & 1.85 & 2.61 & 2.03 & 2.70 & 2.62 & 2.71 & 2.80 & 2.44 & 1.87 & 2.46 & 2.41 & 2.27 \\
\hline
\end{tabular}


Table 1: (Continued)

\begin{tabular}{|c|c|c|c|c|c|c|c|c|c|c|c|c|c|c|c|c|c|c|c|c|}
\hline Rock type & Dacite & & & & & & & & & & Rhyolit & & & & & & & & & \\
\hline Sample No. & GD-5 & GD-6 & GD- 8 & GD-14 & GD-15 & GDP-4 & GDP-7 & GDP-10 & GDP-11 & Average & GD-2 & GD-7 & GD-12 & GD-13 & GD-16 & GD-17 & GD-18 & GDP-20 & GDP-25 & Average \\
\hline $\mathrm{SiO}_{2}$ & 66.17 & 63.60 & 63.12 & 64.65 & 62.35 & 63.79 & 66.03 & 65.54 & 66.88 & 64.64 & 74.13 & 75.81 & 74.09 & 72.48 & 74.42 & 73.68 & 75.39 & 73.41 & 76.42 & 74.43 \\
\hline $\mathrm{TiO}_{2}$ & 0.75 & 0.97 & 0.68 & 0.79 & 0.81 & 0.78 & 0.64 & 0.67 & 0.45 & 0.74 & 0.34 & 0.13 & 0.40 & 0.24 & 0.16 & 0.19 & 0.12 & 0.20 & 0.15 & 0.21 \\
\hline $\mathrm{Al}_{2} \mathrm{O}_{3}$ & 15.67 & 14.68 & 18.09 & 14.36 & 14.77 & 16.12 & 16.55 & 15.54 & 15.36 & 15.69 & 13.24 & 12.51 & 13.67 & 13.89 & 12.78 & 12.96 & 12.74 & 13.91 & 12.51 & 13.13 \\
\hline $\mathrm{Fe}_{2} \mathrm{O}_{3}$ & 3.87 & 6.22 & 5.20 & 5.97 & 6.28 & 6.77 & 4.45 & 5.33 & 3.76 & 5.27 & 1.25 & 1.09 & 1.33 & 1.91 & 1.20 & 1.76 & 1.30 & 1.70 & 0.83 & 1.37 \\
\hline MnO & 0.12 & 0.09 & 0.05 & 0.11 & 0.09 & 0.11 & 0.09 & 0.08 & 0.10 & 0.09 & 0.04 & 0.03 & 0.05 & 0.08 & 0.06 & 0.06 & 0.03 & 0.09 & 0.03 & 0.05 \\
\hline MgO & 1.44 & 2.41 & 1.39 & 2.21 & 2.40 & 1.85 & 1.53 & 1.52 & 1.06 & 1.73 & 0.35 & 0.28 & 0.26 & 0.53 & 0.32 & 0.51 & 0.20 & 0.47 & 0.26 & 0.35 \\
\hline $\mathrm{CaO}$ & 2.50 & 2.83 & 2.53 & 3.09 & 3.49 & 2.51 & 2.26 & 2.38 & 2.31 & 2.64 & 1.06 & 0.31 & 0.69 & 1.23 & 0.90 & 1.17 & 0.57 & 1.04 & 0.41 & 0.82 \\
\hline $\mathrm{Na}_{2} \mathrm{O}$ & 5.24 & 4.73 & 3.93 & 4.65 & 4.38 & 4.08 & 4.39 & 4.39 & 5.11 & 4.65 & 4.57 & 4.39 & 4.29 & 4.18 & 5.16 & 4.56 & 4.79 & 4.62 & 4.88 & 4.60 \\
\hline $\mathbf{K}_{2} \mathrm{O}$ & 2.89 & 2.76 & 3.11 & 2.90 & 2.73 & 3.13 & 3.10 & 3.04 & 3.46 & 3.00 & 3.96 & 4.86 & 4.57 & 4.25 & 3.96 & 3.85 & 3.95 & 3.29 & 3.97 & 4.07 \\
\hline $\mathbf{P}_{2} \mathbf{O}_{5}$ & 0.17 & 0.22 & 0.36 & 0.28 & 0.23 & 0.22 & 0.20 & 0.13 & 0.10 & 0.22 & 0.06 & 0.01 & 0.05 & 0.05 & 0.01 & 0.05 & 0.02 & 0.08 & 0.01 & 0.04 \\
\hline L.O.I & 0.92 & 1.25 & 0.98 & 0.88 & 1.56 & 0.63 & 0.74 & 1.37 & 1.38 & 1.11 & 0.84 & 0.46 & 0.58 & 1.12 & 1.01 & 1.09 & 0.86 & 1.18 & 0.46 & 0.84 \\
\hline Total \% & 99.74 & 99.76 & 99.44 & 99.89 & 99.09 & 99.99 & 99.98 & 99.99 & 99.97 & 99.78 & 99.84 & 99.88 & 99.98 & 99.96 & 99.98 & 99.88 & 99.97 & 99.99 & 99.93 & 99.93 \\
\hline $\mathbf{Q}$ & 17.74 & 16.91 & 21.89 & 18.20 & 16.72 & 20.57 & 22.08 & 21.38 & 18.12 & 18.81 & 29.98 & 30.93 & 29.93 & 28.62 & 27.83 & 29.73 & 31.19 & 31.25 & 31.93 & 30.15 \\
\hline C & 0.00 & 0.00 & 4.52 & 0.00 & 0.00 & 1.98 & 2.34 & 1.01 & 0.00 & 0.99 & 0.00 & 0.00 & 0.53 & 0.30 & 0.00 & 0.00 & 0.00 & 1.05 & 0.00 & 0.21 \\
\hline Or & 17.08 & 16.31 & 18.38 & 17.14 & 16.13 & 18.50 & 18.32 & 17.97 & 20.45 & 17.73 & 23.40 & 28.72 & 27.01 & 25.12 & 23.40 & 22.75 & 23.34 & 19.44 & 23.46 & 24.07 \\
\hline $\mathbf{A b}$ & 44.34 & 40.02 & 33.25 & 39.35 & 37.06 & 34.52 & 37.15 & 37.15 & 43.24 & 39.31 & 38.67 & 37.15 & 36.30 & 35.37 & 43.66 & 38.59 & 40.53 & 39.09 & 41.29 & 38.96 \\
\hline An & 10.70 & 10.67 & 10.20 & 9.74 & 12.58 & 11.02 & 9.91 & 10.96 & 8.75 & 10.41 & 3.92 & 0.07 & 3.10 & 5.78 & 0.01 & 3.52 & 1.59 & 4.64 & 0.50 & 2.57 \\
\hline $\mathbf{D i}$ & 0.00 & 0.00 & 0.00 & 1.12 & 0.60 & 0.00 & 0.00 & 0.00 & 0.68 & 0.24 & 0.00 & 0.83 & 0.00 & 0.00 & 1.72 & 1.19 & 0.62 & 0.00 & 0.83 & 0.58 \\
\hline Hy & 3.59 & 6.00 & 3.46 & 4.99 & 5.70 & 4.61 & 3.81 & 3.79 & 2.32 & 4.19 & 0.87 & 0.31 & 0.65 & 1.32 & 0.00 & 0.72 & 0.21 & 1.17 & 0.27 & 0.61 \\
\hline $\mathrm{Hm}$ & 0.26 & 0.19 & 0.11 & 0.24 & 0.19 & 0.24 & 0.19 & 0.17 & 0.21 & 0.20 & 0.09 & 0.06 & 0.11 & 0.17 & 0.13 & 0.13 & 0.06 & 0.19 & 0.06 & 0.11 \\
\hline Il & 3.87 & 6.22 & 5.20 & 5.97 & 6.28 & 6.77 & 4.45 & 5.33 & 3.76 & 5.27 & 1.25 & 1.09 & 1.33 & 1.91 & 1.20 & 1.76 & 1.30 & 1.70 & 0.83 & 1.37 \\
\hline Tn & 0.42 & 1.36 & 0.00 & 1.64 & 1.74 & 0.00 & 0.00 & 0.00 & 0.83 & 0.63 & 0.67 & 0.24 & 0.00 & 0.00 & 0.23 & 0.30 & 0.21 & 0.00 & 0.29 & 0.21 \\
\hline Ap & 0.40 & 0.52 & 0.85 & 0.66 & 0.54 & 0.52 & 0.47 & 0.31 & 0.24 & 0.53 & 0.14 & 0.02 & 0.12 & 0.12 & 0.02 & 0.12 & 0.05 & 0.19 & 0.02 & 0.09 \\
\hline Ba & 832 & 629 & 532 & 665 & 604 & 952 & 741 & 629 & 891 & 734 & 716 & 715 & 696 & 712 & 518 & 566 & 252 & 654 & 509 & 593 \\
\hline $\mathrm{Cr}$ & 108 & 117 & 60 & 132 & 130 & 92 & 14 & 79 & 102 & 94 & 37 & 55 & 29 & 40 & 54 & 31 & 31 & 37 & 39 & 39 \\
\hline $\mathbf{N i}$ & 18 & 27 & 43 & 32 & 33 & 42 & 6 & 45 & 6 & 27 & 6 & 4 & 3 & 3 & 15 & 3 & 5 & 5 & 6 & 6 \\
\hline $\mathbf{P b}$ & 11 & 8 & 13 & 10 & 8 & 8 & 10 & 11 & 10 & 10 & 15 & 12 & 13 & 12 & 16 & 10 & 13 & 12 & 10 & 12 \\
\hline Ce & 69 & 59 & 57 & 48 & 45 & 47 & 51 & 83 & 65 & 59 & 52 & 71 & 74 & 68 & 51 & 63 & 37 & 43 & 55 & 57 \\
\hline Rb & 50 & 53 & 146 & 55 & 57 & 61 & 62 & 106 & 55 & 69 & 114 & 138 & 108 & 107 & 178 & 88 & 75 & 65 & 93 & 107 \\
\hline Sr & 443 & 659 & 203 & 675 & 726 & 634 & 717 & 295 & 532 & 550 & 276 & 59 & 150 & 185 & 203 & 168 & 124 & 147 & 55 & 152 \\
\hline Nb & 11 & 7 & 10 & 9 & 7 & 8 & 8 & 13 & 9 & 9 & 14 & 24 & 14 & 23 & 37 & 12 & 18 & 21 & 17 & 20 \\
\hline $\mathbf{Y}$ & 28 & 18 & 29 & 16 & 19 & 20 & 19 & 26 & 15 & 21 & 30 & 21 & 25 & 25 & 24 & 22 & 29 & 31 & 28 & 26 \\
\hline $\mathrm{Zr}$ & 274 & 185 & 226 & 247 & 199 & 209 & 243 & 309 & 283 & 243 & 268 & 343 & 239 & 276 & 286 & 265 & 181 & 235 & 254 & 261 \\
\hline Th & 6.2 & 5.4 & 14.3 & 6.0 & 6.0 & 4.7 & 4.5 & 11.9 & 7.7 & 7.2 & 10.2 & 21.0 & 14.0 & 13.0 & 31.0 & 13.1 & 10.2 & 7.4 & 11.4 & 14.6 \\
\hline $\mathbf{U}$ & 1.9 & 1.7 & 6.0 & 1 & 1.8 & 1. & 1 & 3.8 & J & 2.5 & 2.3 & 7.1 & 4.8 & 3.1 & 9.8 & 2.3 & 2.3 & 1.9 & 2.5 & 4.0 \\
\hline K/Rb & 483.34 & 435.62 & 176.72 & 437.74 & 399.02 & 425.29 & 418.47 & 238.10 & 521.32 & 408.01 & 287.88 & 292.37 & 350.65 & 330.99 & 184.70 & 363.63 & 434.92 & 420.21 & 354.40 & 335.53 \\
\hline $\mathbf{R b} / \mathrm{Sr}$ & 0.11 & 0.08 & 0.72 & 0.08 & 0.08 & 0.10 & 0.09 & 0.36 & 0.10 & 0.18 & 0.41 & 2.34 & 0.72 & 0.58 & 0.88 & 0.52 & 0.61 & 0.44 & 1.69 & 0.91 \\
\hline $\mathbf{R b} / \mathbf{Z r}$ & 0.18 & 0.28 & 0.65 & 0.22 & 0.29 & 0.29 & 0.25 & 0.34 & 0.19 & 0.29 & 0.43 & 0.40 & 0.45 & 0.39 & 0.62 & 0.33 & 0.42 & 0.28 & 0.37 & 0.41 \\
\hline $\mathrm{Sr} / \mathbf{Y}$ & 16.11 & 35.80 & 7.14 & 42.19 & 38.20 & 31.54 & 37.35 & 11.44 & 35.47 & 28.43 & 9.30 & 2.27 & 5.98 & 6.59 & 7.52 & 7.69 & 4.29 & 4.75 & 1.99 & 5.60 \\
\hline $\mathbf{Y} / \mathbf{N b}$ & 2.46 & 2.67 & 2.88 & 1.78 & 2.60 & 2.58 & 2.37 & 1.93 & 1.61 & 2.32 & 2.17 & 1.53 & 1.86 & 1.47 & 1.50 & 1.83 & 1.62 & 1.48 & 1.63 & 1.68 \\
\hline
\end{tabular}


Seddiek, et al.

Table 2: Rare earth elements and some geochemical ratios for the studied Dokhan Volcanics.

\begin{tabular}{|c|c|c|c|c|c|c|c|c|c|c|c|c|c|c|c|c|c|c|c|}
\hline \multirow{2}{*}{$\begin{array}{l}\text { Rock type } \\
\text { Sample No. }\end{array}$} & \multicolumn{4}{|c|}{ Basaltic andesite } & \multicolumn{6}{|c|}{ Andesite } & \multicolumn{5}{|l|}{ Dacite } & \multicolumn{4}{|c|}{ Rhyolite } \\
\hline & GDP-2 & GDP-9 & GDP-13 & Average & GD -1 & GD-9 & GDP- 3 & GDP- 8 & GDP -14 & Average & GD-3 & GD-5 & GD-14 & GDP-11 & Average & GD-7 & GD-16 & GDP-25 & Average \\
\hline Hf & 4.0 & 5.1 & 4.3 & 4.5 & 5.2 & 4.7 & 4.5 & 6.8 & 4.9 & 5.2 & 5.7 & 6.8 & 4.0 & 6.8 & 5.8 & 6.1 & 5.3 & 4.8 & 5.4 \\
\hline Ta & 0.4 & 0.4 & 0.4 & 0.4 & 0.4 & 0.5 & 0.6 & 0.6 & 0.7 & 0.6 & 0.5 & 0.7 & 0.7 & 0.7 & 0.6 & 3.2 & 2.6 & 1.1 & 2.3 \\
\hline $\mathbf{L a}$ & 21.19 & 24.52 & 25.10 & 23.60 & 24.52 & 27.09 & 21.18 & 31.77 & 22.52 & 25.42 & 31.42 & 32.50 & 21.30 & 30.00 & 28.81 & 30.75 & 31.62 & 28.90 & 30.42 \\
\hline $\mathrm{Ce}$ & 43.90 & 54.18 & 57.10 & 51.73 & 54.18 & 59.26 & 45.54 & 70.22 & 50.02 & 55.84 & 68.10 & 68.71 & 48.30 & 65.00 & 62.53 & 70.58 & 51.10 & 55.30 & 58.99 \\
\hline Pr & 5.94 & 6.71 & 7.29 & 6.65 & 6.73 & 7.35 & 5.98 & 9.16 & 6.46 & 7.14 & 8.73 & 9.08 & 5.93 & 7.43 & 7.79 & 7.16 & 4.31 & 6.00 & 5.82 \\
\hline Nd & 26.34 & 27.44 & 30.98 & 28.25 & 27.44 & 31.79 & 26.47 & 38.96 & 29.13 & 30.76 & 37.67 & 37.56 & 24.52 & 30.15 & 32.48 & 25.10 & 11.90 & 20.89 & 19.30 \\
\hline Sm & 5.68 & 5.18 & 6.30 & 5.72 & 5.18 & 5.98 & 5.18 & 7.85 & 5.76 & 5.99 & 6.88 & 6.94 & 4.76 & 5.35 & 5.98 & 7.10 & 1.46 & 4.70 & 4.42 \\
\hline Eu & 1.72 & 1.45 & 1.89 & 1.69 & 1.45 & 1.92 & 1.53 & 2.63 & 1.71 & 1.85 & 1.88 & 2.19 & 1.41 & 1.53 & 1.75 & 0.45 & 0.05 & 0.32 & 0.27 \\
\hline Gd & 4.34 & 4.18 & 5.18 & 4.57 & 4.18 & 4.27 & 4.72 & 7.14 & 5.41 & 5.14 & 5.89 & 6.85 & 3.75 & 4.05 & 5.14 & 3.26 & 1.20 & 3.65 & 2.70 \\
\hline Tb & 0.65 & 0.49 & 0.71 & 0.62 & 0.50 & 0.59 & 0.69 & 1.03 & 0.76 & 0.71 & 0.71 & 0.93 & 0.50 & 0.56 & 0.68 & 0.81 & 0.25 & 0.46 & 0.51 \\
\hline Dy & 3.90 & 2.93 & 4.20 & 3.68 & 2.94 & 2.85 & 4.18 & 5.63 & 4.23 & 3.97 & 4.40 & 5.69 & 2.73 & 2.50 & 3.83 & 5.10 & 1.70 & 3.32 & 3.37 \\
\hline Ho & 0.72 & 0.51 & 0.74 & 0.66 & 0.52 & 0.68 & 0.83 & 1.05 & 0.81 & 0.78 & 0.71 & 0.92 & 0.50 & 0.54 & 0.67 & 1.00 & 0.42 & 0.65 & 0.69 \\
\hline Er & 1.92 & 1.37 & 2.00 & 1.76 & 1.36 & 1.71 & 2.17 & 2.99 & 2.40 & 2.13 & 1.80 & 2.77 & 1.23 & 1.51 & 1.83 & 3.75 & 1.46 & 1.80 & 2.34 \\
\hline Tm & 0.27 & 0.16 & 0.31 & 0.25 & 0.17 & 0.22 & 0.33 & 0.42 & 0.31 & 0.29 & 0.24 & 0.39 & 0.18 & 0.26 & 0.27 & 0.39 & 0.31 & 0.25 & 0.32 \\
\hline Yb & 2.10 & 1.24 & 1.98 & 1.77 & 1.24 & 1.21 & 2.18 & 2.88 & 2.15 & 1.93 & 2.10 & 2.60 & 0.99 & 1.73 & 1.86 & 2.87 & 2.30 & 1.66 & 2.28 \\
\hline Lu & 0.36 & 0.15 & 0.33 & 0.28 & 0.16 & 0.23 & 0.33 & 0.48 & 0.32 & 0.30 & 0.20 & 0.39 & 0.17 & 0.21 & 0.24 & 0.43 & 0.44 & 0.29 & 0.39 \\
\hline LaN/LuN & 6.11 & 16.97 & 7.89 & 10.32 & 15.91 & 12.23 & 6.66 & 6.87 & 7.31 & 9.80 & 16.32 & 8.65 & 13.01 & 14.84 & 13.21 & 7.42 & 7.47 & 10.35 & 8.41 \\
\hline LaN/YbN & 6.80 & 13.33 & 8.54 & 9.56 & 13.33 & 15.09 & 6.55 & 7.43 & 7.06 & 9.89 & 10.09 & 8.43 & 14.51 & 11.69 & 11.18 & 7.22 & 9.27 & 11.74 & 9.41 \\
\hline $\mathrm{LaN} / \mathrm{SmN}$ & 2.34 & 2.97 & 2.50 & 2.60 & 2.98 & 2.85 & 2.57 & 2.55 & 2.45 & 2.68 & 2.87 & 2.95 & 2.81 & 3.53 & 3.04 & 2.72 & 13.62 & 3.86 & 6.73 \\
\hline GdN/LuN & 1.5 & 3.46 & 1.95 & 2.30 & 3.25 & 2.31 & 1.78 & 1.85 & 2.10 & 2.26 & 3.66 & 2.18 & 2.74 & 2.40 & 2.75 & 0.94 & 0.34 & 1.56 & 0.95 \\
\hline $\mathbf{E u} / \mathbf{E u} u^{*}$ & 1.06 & 0.95 & 1.01 & 1.01 & 0.95 & 1.16 & 0.95 & 1.07 & 0.94 & 1.01 & 0.90 & 0.97 & 1.02 & 1.01 & 0.98 & 0.28 & 0.11 & 0.23 & 0.21 \\
\hline
\end{tabular}


Petrological and geochemical investigations on Dokhan Volcanics

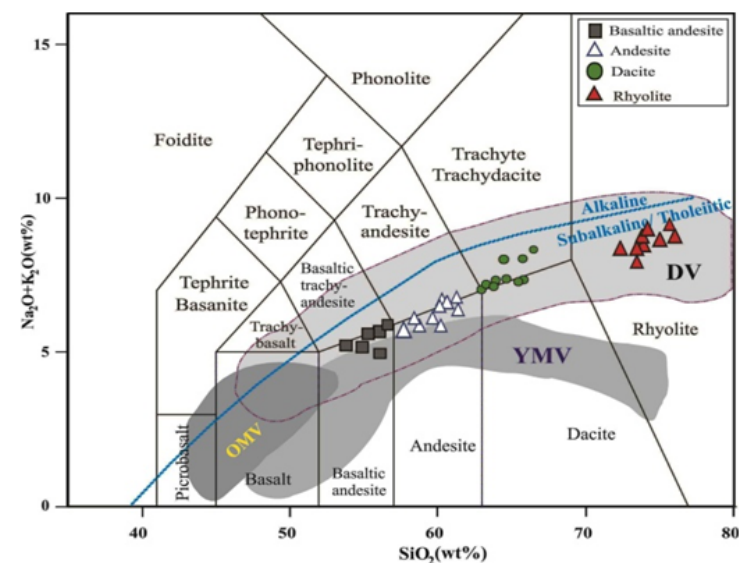

Fig. 4: TAS chemical classification diagram after Le Bas et al. (1986) for the studied volcanics. The dividing curve between subalkaline and alkaline rocks after Irvine and Baragar (1971). Fields of Older and Younger metavolcanics (OMV and YMV) from Stern (1981), and Dokhan Volcanics (DV) from other areas (Basta et al., 1980; Ressetar and Monard, 1983; Stern and Gottfried, 1986; Abdel Rahman, 1996; Eliwa, 2000; Moghazi 1994, 2003).

Plots of $\mathrm{SiO}_{2}$ vs. some selected trace elements concentrations are illustrated in Fig. 5. Cr and Ni decrease in volcanics lavas from basaltic andesite to rhyolite, whereas $\mathrm{Rb}, \mathrm{Nb}, \mathrm{Y}$, and $\mathrm{Zr}$ slightly increase or remain constant with increasing $\mathrm{SiO}_{2}$ content, while $\mathrm{Sr}$ content show negative correlation with increasing $\mathrm{SiO}_{2}$ values. $\mathrm{Ba}$ show gradual increase in their contents up to a maximum value of approximately 72 wt. \% $\mathrm{SiO}_{2}$ after which a sharp decrease occurs with increasing $\mathrm{SiO}_{2}$ which may be ascribed to fractionation of $\mathrm{K}$-feldspar or biotite in the evolved volcanics. This behavior of $\mathrm{Ba}$, also observed in other fractionating calc-alkaline DokhanVolcanics, documented by several authors (e.g. Mohamed et al., 2000; Moghazi, 2003; Eliwa et al., 2006; El-Bialy, 2010; Be'eri-Shlevinet al., 2011). This feature is common in calc-alkaline igneous suites (Miller and Mittlefehldt 1984; Abdel Rahman 1996) where the sharp decrease in $\mathrm{Al}_{2} \mathrm{O}_{3}$ and $\mathrm{Ba}$ can be attributed to the dominant role of feldspar fractionation.

Fig.5: Variation diagrams showing the distribution of trace elements (ppm) vs. SiO2wt.\% for the studied volcanics.
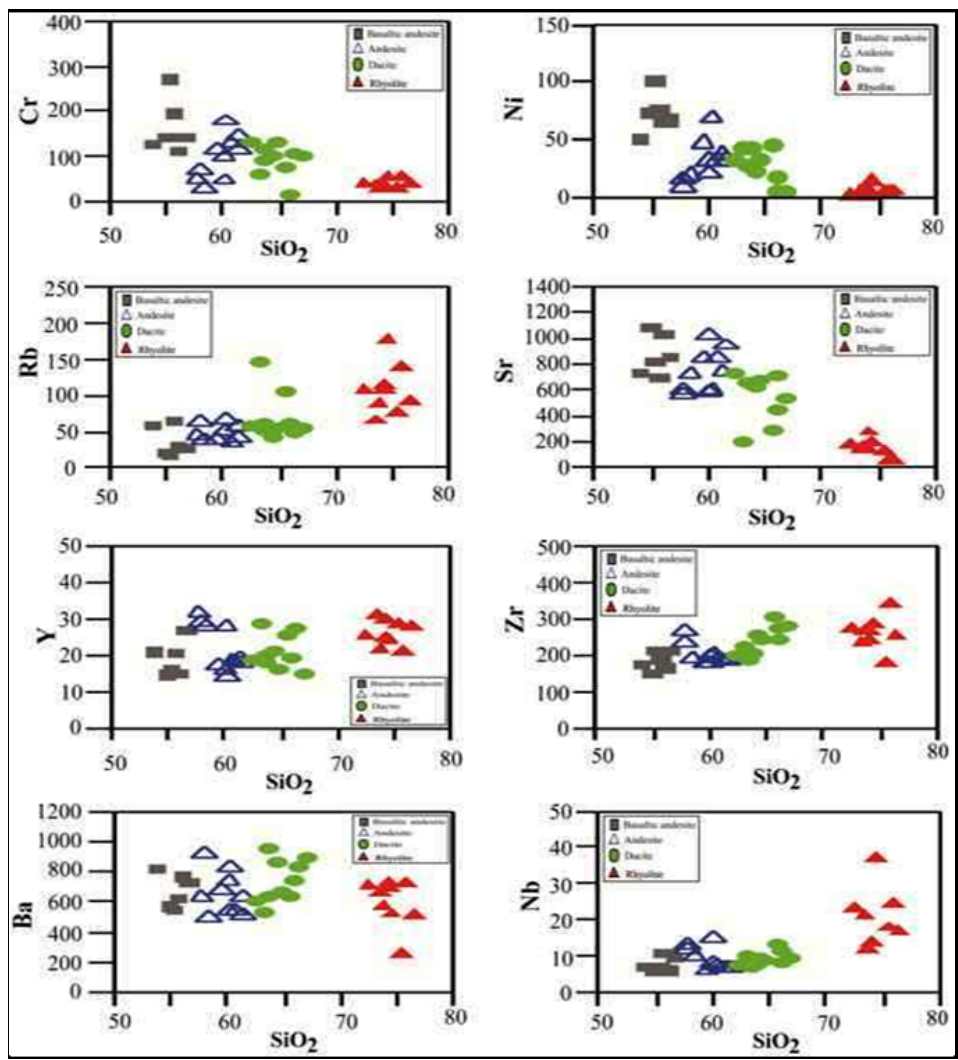

The decrease in $\mathrm{Zr}$ has been attributed to early crystallization of zircon due to decreasing solubility of $\mathrm{Zr}$ in a melt with low alkali/aluminum ratio (Watson 1979).

Table 1 reveals that the studied DokhanVolcanics exhibit a significantly wide range of $\mathrm{Rb} / \mathrm{Sr}$ ratios $(0.02-2.34)$ with an average of 0.31 . The average as well as many of these $\mathrm{Rb} / \mathrm{Sr}$ values are rather higher than lower and middle continental crust which have $\mathrm{Rb} / \mathrm{Sr}$ ratios of 0.12 and 0.22 respectively (Rudnick and Fountain, 1995;Wedepohl, 1995). Such elevated $\mathrm{Rb} / \mathrm{Sr}$ ratios point to a 


\section{Seddiek, et al.}

role of the continental crust in the genesis of these volcanics, since the crust can be regarded as being vertically zoned with $\mathrm{Rb} / \mathrm{Sr}$ increasing upward (Blevin and Chappell, 1995).

Additionally, $\mathrm{Rb}$ and $\mathrm{Sr}$ display fair correlation (Fig. 5) with increasing $\mathrm{SiO}_{2}$ values indicating that the magmatic abundances of these elements were not significantly modified during alteration and metamorphism. This is supported by the systematic correlation between $\mathrm{K}_{2} \mathrm{O}$ and $\mathrm{Rb}$, which implies that the $\mathrm{K} / \mathrm{Rb}$ ratios were not modified by post-magmatic processes. The $\mathrm{K} / \mathrm{Rb}$ ratios of the studied volcanic samples range between 176 and 820 (Table 1), and many of them surpass crustal rocks (150350; Taylor, 1965) and the chondritic values (242; Anders and Grevesse, 1989). However, such high $\mathrm{K} / \mathrm{Rb}$ ratios indicate that magmatic processes have absolutely controlled the evolution of these volcanics with no involvement of post-magmatic aqueous fluid phase inter-actions or mineral growth in the presence of aqueous fluids, which typically produce $\mathrm{K} / \mathrm{Rb}$ ratios less than 100 (Shearer et al., 1985;Bau, 1997; Irber, 1999). Y/Nb ratios of the studied volcanics vary between a minimum of 1.47 and a maximum of 2.96 (Table .1).

Eby $(1990,1992)$ has shown that magmas derived from mantle sources have $\mathrm{Y} / \mathrm{Nb}$ ratios less than 1.2 , while $\mathrm{Y} / \mathrm{Nb}$ ratios greater than 1.2 characterize rocks of crustal origin. Therefore, this ratio can be considered so crucial indentifying the source of magma. Accordingly, as the $\mathrm{Y} / \mathrm{Nb}$ ratios of all of the studied Dokhan Volcanic samples (1.47-2.96) are above the 1.2 discriminate value, they are undoubtedly entirely derived from crustal sources with little or no mantle contribution.

\section{Crustal contamination}

It is important to assess first whether or not the studied Dokhan volcanics have undergone crustal contamination. This is because mafic rocks are absent and most of the rock varieties are porphyritic indicating that they might have resided in crustal magma chambers prior to eruption and would thus had adequate residence time to interact with continental crust. For this purpose, evidences from several trace element ratios for the investigated volcanics are considered. The LILE ( $\mathrm{Rb}, \mathrm{Ba}$ and $\mathrm{K}$ ) and $\mathrm{Zr}$ are incompatible with respect to the main crystallizing phenocryst phases (e.g. plagioclase, augite and hornblende) in the present lavas (Green, 1980). The ratios of such incompatible elements like K/Rb, $\mathrm{Ba} / \mathrm{Nb}, \mathrm{Y} / \mathrm{Nb}$ and $\mathrm{Rb} / \mathrm{Zr}$ do not significantly change by simple fractional crystallization of this assemblage. Variations in these ratios are preferably related to crustal contamination by assimilation fractional crystallization processes (Davidson et al., 1987 \& 1988).

The Dokhan volcanic rocks (Figs. 6 a\&b) show that, most of the samples of the intermediate volcanics have no significant variation in the $\mathrm{Ba} / \mathrm{Nb}$ and $\mathrm{Y} / \mathrm{Nb}$ ratios indicating closed-system evolution processes. The recognition of $\sim 680 \mathrm{Ma}$ xenocrysts in andesite samples of the Dokhan Volcanics at the type locality area at Gebel Dokhan (Wilde and Youssef, 2000) support the idea of crustal contamination during evolution of these rocks. In the felsic volcanics, there are wider ranges in the $\mathrm{Ba} / \mathrm{Nb}$ and $\mathrm{Y} / \mathrm{Nb}$ values, which indicate that significant contamination is involved at the very end of evolution of the entire volcanic suite.

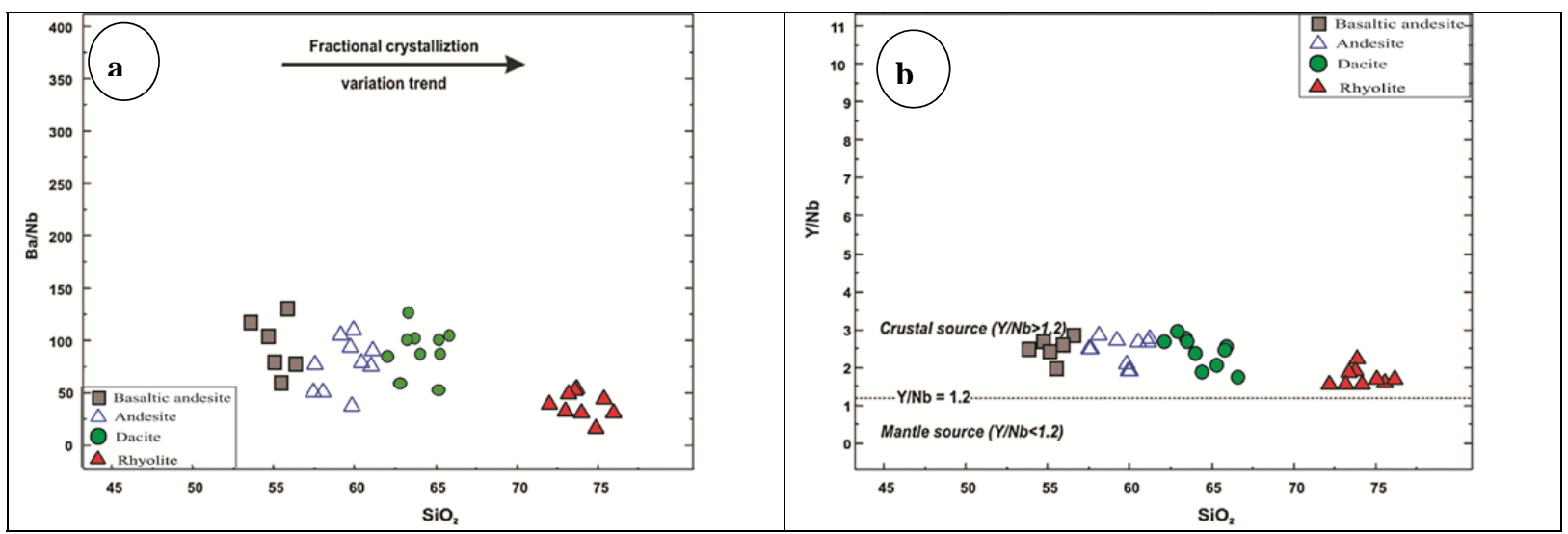

Fig. 6: (a) $\mathrm{SiO}_{2}$ vs. $\mathrm{Ba} / \mathrm{Nb}$, and (b) $\mathrm{SiO}_{2}$ vs. $\mathrm{Y} / \mathrm{Nb}$ diagrams for the studied Volcanics showing the variation of these ratios with increasing $\mathrm{SiO}_{2}$. The $\mathrm{Y} / \mathrm{Nb}$ discriminate value at 1.2 in (b) is after Eby (1990). 


\section{Magma type}

On the $\mathrm{K}_{2} \mathrm{O}$ versus $\mathrm{SiO}_{2}$ variation diagram of Le Maitre et al. (1989), the studied Dokhan volcanics are classified into medium- to high-K varieties (Fig. 7) with more high-K affinities in evolved rhyolite lavas. The alumina saturation index (ASI) [molar ratio $\mathrm{Al}_{2} \mathrm{O}_{3} / \mathrm{CaO}+\mathrm{Na}_{2} \mathrm{O}+\mathrm{K}_{2} \mathrm{O}$ ] of the studied volcanic samples covers a wide range $\mathrm{ASI}=0.74-1.25$ (Table 1 ), reflecting the metaluminous to slightly peraluminous character of the suite. They all have agpaitic index ( $\mathrm{AI}$ ) $\left[\left(\mathrm{Na}_{2} \mathrm{O}+\mathrm{K}_{2} \mathrm{O}\right) / \mathrm{Al}_{2} \mathrm{O}_{3}\right.$ molar] less than 0.87 (Table 1), characteristic of calc-alkaline rocks (Liégeois and Black, 1987; Liégeois et al., 1998), but few samples of rhyolite have agpaitic index more than 0.87 , which indicate increasingly alkaline nature with increasing degree of differentiation.

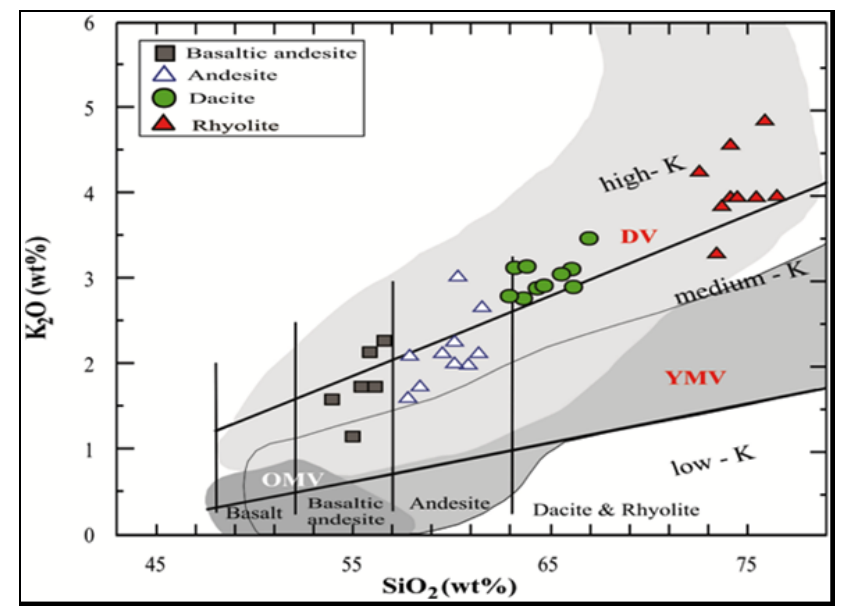

Fig. 7: $\mathrm{SiO}_{2}$ vs. $\mathrm{K}_{2} \mathrm{O}$ discrimination diagram for the studied volcanic after Le Maitre et al. (1989). Fields for OMV, YMV, and DV as in fig. 3.

\section{Rare Earth Elements (REE) Patterns}

The concentrations of rare earth elements (REE) of the studied volcanics, along with the Eu anomaly measure $\left(\mathrm{Eu} / \mathrm{Eu}^{*}\right)$ and some significant normalized REE's ratios (Sun and McDonough, 1989) are given in Table (2). The rare earth element contents of the studied volcanics are plotted as chondrite-normalized REE patterns in (Fig. 8). The Dokhan volcanic rocks have LREE-enriched chondrite -normalized REE patterns, which are generally parallel to subparallel LREE-enriched with rather decrease from $\mathrm{La}$ to $\mathrm{Eu}$. These volcanics are characterized by moderate degree of REE fractionation, $\left[(\mathrm{La} / \mathrm{Yb})_{\mathrm{N}}=6.8-15.09\right]$. The degree of REE fractionation slightly increases from basaltic andesite $\left[(\mathrm{La} / \mathrm{Yb})_{\mathrm{N}}=6.8-13.33\right]$ to andesite $\left[(\mathrm{La} / \mathrm{Yb})_{\mathrm{N}}=6.55-15.09\right]$. On the other hand, the dacites lavas have slightly fractionated REE patterns with more elevated light LREE and depleted heavy HREE than those in basaltic andesites, whereas the degree of REE fractionation in dacites $\left[(\mathrm{La} / \mathrm{Yb})_{\mathrm{N}}=8.43\right.$ 14.51] is comparable to those of the andesites, implying that the dacites are not fractionated from the same magma produced the andesites. The intermediate volcanics are characterized by low fractionated LREE $\left[(\mathrm{La} / \mathrm{Sm})_{\mathrm{N}}=2.34-2.98\right]$ and rather flat heavy REE patterns $\left[(\mathrm{Gd} / \mathrm{Lu})_{\mathrm{N}}=1.55-3.46\right]$. These rocks have low $\Sigma$ REE content (119-182ppm), often considered indicative of garnet in the source (Castillo, 2012). The REE patterns of the rhyolites are characterized by U-shaped with strong negative Eu anomaly $\left(\mathrm{Eu} / \mathrm{Eu}^{*}=0.11-0.28\right)$. Negative $\mathrm{Eu}$ anomaly in the felsic lavas suggests fractionation of hornblende with plagioclase from basaltic andesite of the same suite.

Fig. 8: Chondrite normalized REE patterns of the studied volcanics.

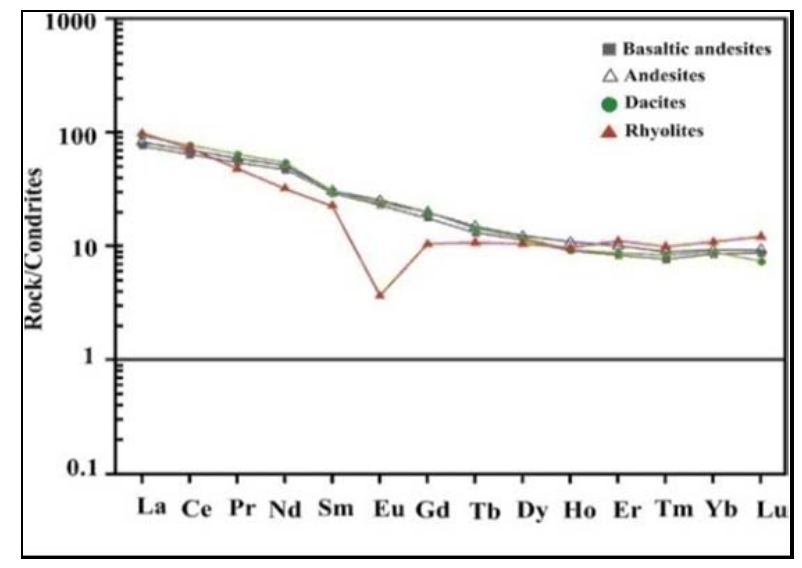




\section{Tectonic Setting}

The studied Dokhan volcanic rocks are essentially medium- to high-K intermediate to felsic rocks. These volcanic rocks share geochemical characteristics common in subduction-related and postcollisional magmas, such as enrichment of LILE (Saunders et al., 1980; Pearce, 1983; Wilson, 1994; Hollings and Wyman, 1999; Gill, 2010), and similar to those of rocks developed in volcanic arc setting. The tectonic setting for the studied Dokhan Volcanicsis achieved throughout a number of conventional discrimination diagrams based on whole-rock chemistry of immobile elements (e.g., $\mathrm{Nb}$, $\mathrm{Ti}, \mathrm{Y}, \mathrm{Rb}$ and $\mathrm{Zr}$ ). The $\mathrm{Zr}$ (ppm) versus $\mathrm{SiO}_{2}$ (wt.\%) discrimination diagram of Ewart (1982). Fig. 9 shows that, the studied Dokhan volcanics plot in the active continental margin field.

On the $\mathrm{Th} / \mathrm{Yb}$ vs. $\mathrm{Ta} / \mathrm{Yb}$ (Gorton and Schandal, 2000) diagram, the studied intermediate-felsic volcanic rocks plotting in the active continental margin field (Fig. 10). This diagram mostly indicates that, the Dokhan Volcanics erupted in an arc environment with thicker (continental) crust. The studied volcanic samples are characterized by strong enrichment of large ion lithophile elements (LILEs; e.g. $\mathrm{K}_{2} \mathrm{O}, \mathrm{Rb}, \mathrm{Ba}, \mathrm{Sr}, \mathrm{Th}$ ) relative to high field strength elements (HFSEs; i.e. $\mathrm{Nb}, \mathrm{Zr}, \mathrm{Y}, \mathrm{Yb}$ ) (Table .1), a typical feature of continental-arc rocks formed through active margin-related processes (Pearce et al., 1984; Grove et al., 2003). The relatively high $\mathrm{K}_{2} \mathrm{O}$ (3.01-1.14wt \%), Ba (913-495ppm) and $\mathrm{Sr}(1088-$ $570 \mathrm{ppm})$ contents and also the high ratios of $\mathrm{Zr} / \mathrm{Y}(6.35-14.5)$ of the basaltic andesite and andesite samples are all likewise typical of calc-alkaline magmas formed in continental arcs (Wilson, 1989, 1994; Pearce, 1983).

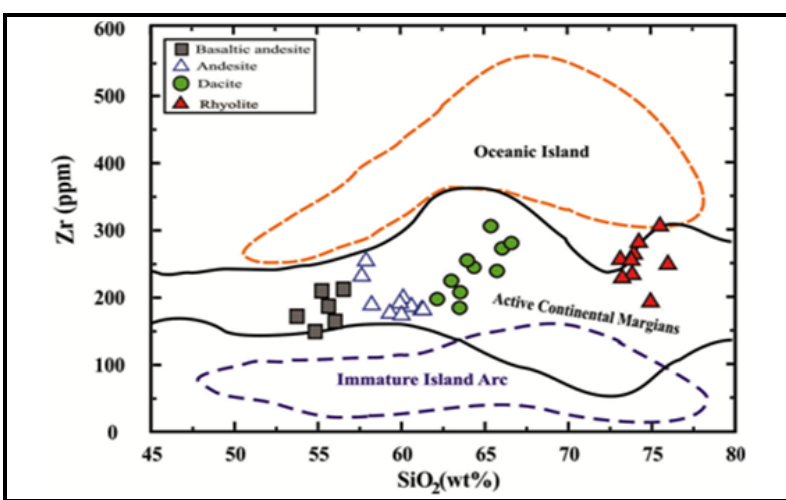

Fig. 9: $\mathrm{Zr}(\mathrm{ppm})$ versus $\mathrm{SiO}_{2}$ (wt.\%) diagram for the studied volcanics (Ewart ,1982).

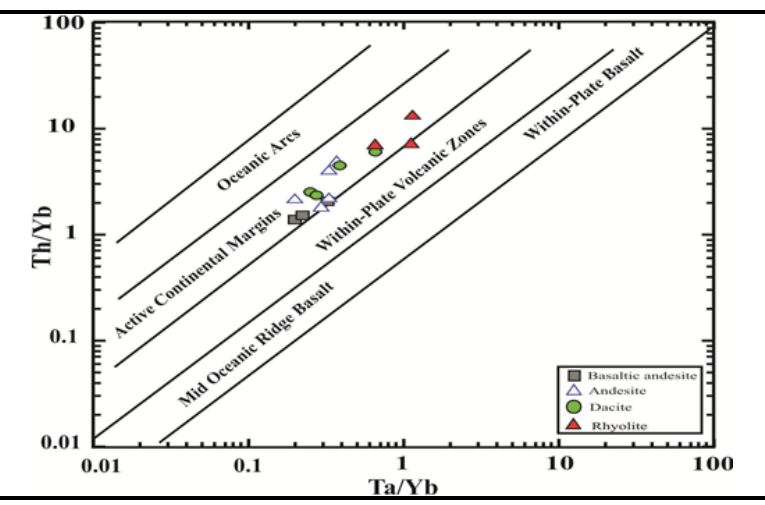

Fig. 10: Tectonic discrimination diagram of $\mathrm{Th} / \mathrm{Yb}$ vs. $\mathrm{Ta} / \mathrm{Yb}$ for the studied volcanics after Gorton and Schandal (2000).

We can conclude that the Dokhan volcanic rocks were generated in subduction setting rather than transitional or post-collisional setting (Ressetar and Monrad, 1983; Stern et al., 1984; Moghazi, 2003; Eliwa et al., 2006; El-Bialy, 2010; Eliwa et al., 2014; Obeid and Azer, 2015). The arc-related geochemical characteristics suggest that subduction continued until near end of the collision stage beneath the Dokhan Volcanics province, or that the mantle source retained a geochemical memory of subduction-related modifications. In summary, the Dokhan Volcanics erupted during the transition from subduction-related magmatism to extension-related magmatism; slightly after the terminal collision between E and W Gondwana occurred and starting the extension.

\section{PETROGENESIS}

\section{Fractional crystallization}

Several petrographic and geochemical observations provide fundamental constraints on the petrogenesis of the Dokhan Volcanics. The close spatial association of the various volcanic rock varieties suggests that these volcanics are genetically related. The systematic variation of the major and trace elements of the investigated Dokhan Volcanic samples can be interpreted in terms of fractional crystallization. This is implied from the curvilinear trends of some elements, especially $\mathrm{MgO}$ and $\mathrm{Rb}$, and the turnover of the trends of $\mathrm{Al}_{2} \mathrm{O}_{3}, \mathrm{Ba}$, and $\mathrm{Zr}$ (Figs. 5\&6) as a result of changing the fractionating assemblage. Further, this fractionation is evidenced by the variation of major oxides and trace element abundances along continuos trends of decreasing $\mathrm{Al}_{2} \mathrm{O}_{3}, \mathrm{CaO}, \mathrm{MgO}, \mathrm{Fe}_{2} \mathrm{O}_{3}, \mathrm{TiO}_{2}$, $\mathrm{P}_{2} \mathrm{O}_{5}, \mathrm{Ni}, \mathrm{Cr}$ and $\mathrm{Sr}$ and increasing $\mathrm{K}_{2} \mathrm{O}, \mathrm{Rb}, \mathrm{Nb}$ and $\mathrm{Zr}$ (Figs. 5 and 6) with increasing $\mathrm{SiO}_{2}$. 
These trends are qualitatively consistent with the systematic changes in mineralogy, especially the phenocrysts assemblages; with increasing $\mathrm{SiO}_{2}$ from the basaltic andesites to the rhyolites. The oscillatory behavior of alumina against silica has suggested that the amphiboles were the major fractionating mineral assemblage in andesites, whereas plagioclase minerals were the main fractionating phases both as early in basaltic andesites and later from dacites to high-silica rhyolites (Fig. 5). The reversal behavior of $\mathrm{Ba}$ with differentiation from continuous rise to decline in the high$\mathrm{SiO} 2$ lavas may be due to fractionation of $\mathrm{K}$-feldspar in the evolved volcanics (Fig. 6). The relative depletion in $\mathrm{P}$ and Ti relative to MORB composition is most plausibly linked to crystal fractionation of apatite and ilmenite during magmatic evolution.

The similarity and parallel nature of the normalized REE patterns (Fig. 8) with increasing total REE contents and decreasing $\mathrm{Eu} / \mathrm{Eu} *$ with increasing $\mathrm{SiO}_{2}$ imply a major role of crystal fractionation during the evolution of these rocks. Also, the pronounced negative Eu anomalies in the REE patterns of rhyolites lavas require extensive fractionation of plagioclase feldspar.

By using the $\mathrm{Tb} / \mathrm{Yb}$ vs. Yb (ppm) diagrams (Fig. 11a), the studied Dokhan volcanics exhibit varied $\mathrm{Yb}(\mathrm{ppm})$ with nearly constant $\mathrm{Ta} / \mathrm{Yb}$ ratios, sub-paralleling the fractional crystallization (FC) trend (Fan et al., 2004). However, in the $\mathrm{Tb} / \mathrm{Yb}$ vs. $\mathrm{Yb}(\mathrm{ppm})$ diagram the samples exhibit very slight negative trend, which may be due to some kind of source heterogeneity even FC processes were the major controlling factor in the petrogenesis of these lavas. The major role of fractional crystallization in the genesis of these lavas is considered using the $\mathrm{Th} / \mathrm{Nb}$ elemental ratios plotted against $\mathrm{Zr}(\mathrm{ppm})$. Such a plot permits the distinction between magmatic evolutions controlled by simple fractional crystallization in closed systems (that produce an almost horizontal trend with increasing fractionation), and those affected by additional processes. In this diagram, the studied samples define a general horizontal trend characterized by constrained range of $\mathrm{Th} / \mathrm{Nb}$ ratios (Fig.11b), suggesting that fractional crystallization is certainly the most effective process during magmatic evolution.

The above-mentioned characteristics confirm previous suggestions that the Dokhan Volcanics evolved mainly through fractional crystallization of the plagioclase + augite + hornblende + magnetite in the intermediate Dokhan volcanic rocks and plagioclase + K-feldspar + biotite + apatite + magnetite in the acidic varieties.

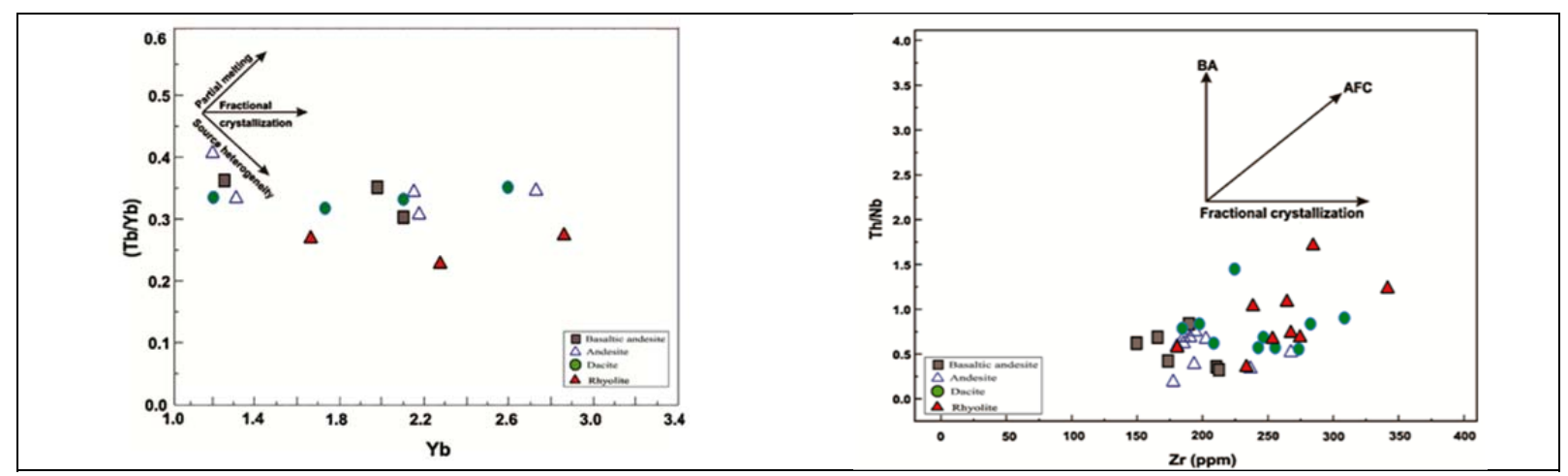

Fig.11: (a) $\mathrm{Yb}$ vs. $\mathrm{Tb} / \mathrm{Yb}$ (b) and $\mathrm{Zr}$ vs. $\mathrm{Th} / \mathrm{Nb}$ diagrams of the studied Volcanics. The trends identifying processes in (a) are after Fan et al. (2004). Schematic trends, in (b), reflecting increasing fractional crystalliza-tion (FC), assimilation-fractional crystallization (AFC), and bulk assimilation (BA) are from Nicolae and Saccani (2003).

\section{SUMMARY AND CONCLUSIONS}

The Dokhan volcanic rocks are exposed in the central (Gabal Dokhan) and the eastern parts of the mapped area. They are represented by a thick sequence of stratified lava flows of basaltic andesites, andesites, dacites, and rhyolites with pyroclastics which are represented by tuffs, agglomerates and ignimbrite. The Dokhan volcanics are intruded by monzogranites, syenogranites and alkali feldspar granites with sharp and irregular contacts, and sometimes are represented by large apophyses and several offshoots in syenogranites and as roofpendant over the monzogranites.

Dokhan volcanics are metaluminous to slightly peraluminous, medium to high-K calc- alkaline affinities and are unmetamorphosed with dominantly porphyritic texture and they enriched in LREE. The REE patterns of the rhyolites are characterized by wing shape with strong negative Eu anomaly, 
Seddiek, et al.

reflecting plagioclase fractionation. The studied Dokhan volcanic rocks have geochemical characteristics similar to the rocks developed in volcanic arc setting in subduction environment rather than transitional or post-collisional setting. Rhyolites have geochemical characteristics of lavas erupted in collisional setting.

The systematic variation of the major and trace elements of the investigated Dokhan Volcanic samples can be interpreted in terms of fractional crystallization. This is evidenced by the variation of major oxides and trace element abundances along continuos trends of decreasing $\mathrm{Al} 2 \mathrm{O} 3, \mathrm{CaO}, \mathrm{MgO}$, $\mathrm{Fe} 2 \mathrm{O} 3, \mathrm{TiO} 2, \mathrm{P} 2 \mathrm{O} 5, \mathrm{Ni}, \mathrm{Cr}$ and $\mathrm{Sr}$ and increasing $\mathrm{K} 2 \mathrm{O}, \mathrm{Rb}, \mathrm{Nb}$ and $\mathrm{Zr}$ with increasing $\mathrm{SiO}_{2}$. The similarity and parallel nature of the normalized REE patterns with increasing total REE contents and decreasing $\mathrm{Eu} / \mathrm{Eu} *$ with increasing $\mathrm{SiO}_{2}$ imply a major role of crystal fractionation during the evolution of these rocks. Also, the pronounced negative Eu anomalies in the REE patterns of rhyolites lavas require extensive fractionation of plagioclase feldspar.

\section{REFERENCES}

Abdel-Rahman, A. M., (1996): Pan-African volcanism: petrology and geochemistry of the Dokhan Volcanic suite in the northern Nubian Shield. Geol. Mag. 133, 17-31.

Abdel-Rahman, A.M., and Doig, R., (1987): The Rb-Sr geochronological evolution of the Ras Gharib segment of the northern Nubian Shield. J. Geol. Soc. Lond. 144, 577-586.

Abdel-Wahed, A. A. A., Ali, K. G., Khalil, M. M. A., Abdel Gawad, A. E., (2012): Dokhan volcanics of Gabal Monqul area, North Eastern Desert, Egypt: geochemistry and petrogenesis. Arab J. Geosci. 5, 29-44.

Akaad, M. K., and Noweir, A. M., (1980): Geology and lithostratigraphy of the Arabian Desert orogenic belt of Egypt between latitudes $25^{\circ} 35 \mathrm{~N}$ and $26^{\circ} 30 \mathrm{~N}$. Inst. Appl. Geol. Jeddah Bull. $3,127-135$.

Anders, E., and Grevesse, N., (1989): Abundances of the elements: Meteoritic and solar. Geochim. Cosmochim. Acta 53, 197-214.

Azer, M. K. and Farahat, E. S., (2011): Late Neoproterozoic volcano-sedimentary succession of Wadi Rufaiyil, southern Sinai, Egypt: a case of transition from late- to post-collisional magmatism. J. Asian Earth Sci., 42, 1187-1203.

Basta, E.Z., Kotb, H. and Awadalla, M.F. (1980): Petrochemical and geochemical characteristics of the Dokhan Formation at the type locality, Jabal Dokhan, Eastern Desert, Egypt. Institute of Applied Geology of Jeddah Bulletin 3, 121-140.

Bau, M., (1997): The lanthanide tetrad effect in highly evolved felsic igneous rocks-Areply to the comment by Y Pan. Contrib. Mineral. Petrol. 128, 409-412.

Be'eri-Shlevin, Y., Eyal, M., Eyal, Y., Whitehouse, M. J. and Litvinovsky, B., (2012): The Sa'alvolcano-sedimentary complex (Sinai, Egypt): a latest Mesoproterozoic volcanicarc in the northern Arabian Nubian Shield. Geology 40, 403-406.

Be'eri-Shlevin, Y., Katzir, Y., Whitehouse, M. J. and Kleinhanns, I. C., (2009): Contribution of pre Pan- African crust to formation of the Arabian Nubian Shield: newsecondary ionization mass spectrometry U-Pb and O studies of zircon. Geology, 37, 899-902.

Be'eri-Shlevin, Y., Samuel, M. D., Azer, M. K., Rämö, O.T., Whitehouse, M. J. and Moussa, H. E., (2011): The late Neoproterozoic Ferani and Rutig volcano-sedimentary successions of the northernmost Arabian-Nubian Shield (ANS): New insights from zircon U-Pb geochronology, geochemistry and O-Nd isotope ratios. Precamb. Res., 188, 21-44.

Blevin, P. L., and Chappell, B. W. (1995): Chemistry, origin and evolution of mineralized granites in the Lachlan Fold Belt Australia: the metallogeny of I- and S-type granites. Econ. Geol. 90, 16041619.

Breitkreuz, C., Eliwa, H., Khalaf, I., El Gameel, K., Bühler, B., Sergeev, S., Larionov, A. and Murata, M. (2010): Neoproterozoic SHRIMP U-Pb zircon ages of silica-rich Dokhan Volcanics in the North Eastern Desert, Egypt. Precambrian Research 182, 163-174.

Castillo, P.R., (2012): Adakite petrogenesis. Lithos 134-135, 304-316.

Davidson, J. P., Dungan, M. A., Ferguson, K. M., and Colucci, M. T. (1988): Crust-magma interactions and the evolution of arc magmas: the San Pedro-Pellado Volcanic complex, southern Chilean Andes. Geology 15:443-446. 
Petrological and geochemical investigations on Dokhan Volcanics

Davidson, J. P., Ferguson, K. M., Colucci, M. T., and Dungan, M. A., (1987): The origin of magmas from the San Pedro-Pellado Volcanic complex, S. Chile: multicomponent sources and open system evolution. Contrib. Mineral Petrol 100:429-445.

Eby, G. N., (1990): The A-type granitoids: a review of their occurrence and chemical characteristics and speculations on their petrogenesis. Lithos, 26, 115-134.

Eby, G. N., (1992): Chemical subdivision of the A-type granitoids: petrogenetic and tectonic implications. Geology 20, 641-644.

El-Bialy, M. Z., (2010): On the Pan-African transition of the Arabian-Nubian Shield from compression to extension: The post-collision Dokhan volcanic suite of Kid-Malhak region, Sinai, Egypt. Gondwana Res., 17, 26-43.

El-Gaby, S., (1975): Petrochemistry and geochemistry of some granites from Egypt. N. Jb. Miner. Abh. 124, 89-148.

El-Gaby, S., Khudeir, A.A., Abdel Tawab, M., and Atalla, R.F., (1991): The metamorphosed volcanosedimentary succession of Wadi Kid, Southeastern Sinai, Egypt. Ann. Geol. Surv. Egypt 17:19-35

Eliwa, H. A., (2000): Petrology, geochemistry, mineral chemistry and petrogenesis of Samr El-Qaa Volcanics, North Eastern Desert, Egypt. Sci. J. Fac. Sci. Minufiya Univ. XIV, 1-45.

Eliwa, H. A., Breitkreuz, C., Khalaf, M.I. and El Gameel, K., (2010): Depositional styles of Early Ediacaran terrestrial volcano-sedimentary succession in Gebel El Urf area, North Eastern Desert, Egypt. J. Afr. Earth Sci. 57, 328-344.

Eliwa, H. A., El-Bialy, M. Z. and Murata, M., (2014): Ediacaran post-collisional volcanism in the Arabian-Nubian Shield: The high-K calc-alkaline Dokhan Volcanics of Gabal Samr El-Qaa (592 \pm $5 \mathrm{Ma}$ ), North Eastern Desert, Egypt. Precambrian Research 246, 180-207.

Eliwa, H. A., Kimura, J.-I., and Itaya, T., (2006): Late Neoproterozoic Dokhan Volcanics, North Eastern Desert, Egypt: Geochemistry and petrogenesis. Precambrian Research 151, 31-52.

El-Sayed, M. M., Obeid, M. A., Furnes, H. and Moghazi, A. M., (2004): Late Neoproterozoic volcanism in the southern Eastern Desert, Egypt: petrological, structural and geochemical constraints on the tectonic-magmatic evolution of the Allaqi Dokhan volcanic suite. Neues Jahrbuch Mineralogie Abhandlungen, 180, 261-286.

Ewart, A., (1982): The mineralogy and petrology of Tertiary-Recent orogenic volcanic rocks with special reference to the andesitic-basaltic composition range. In: Thorpe, R. S. (ed), Andesites. Wiley, Chichester, 25 - 87.

Fan, W. M., Guo, F., Wang, and Y. J. and Zhang, M., (2004): Late Mesozoic volcanism in the northern Huaiyang tectono-magmatic belt, central China: partial melts from alithospheric mantle with subducted continental crust relicts beneath the Dabieorogen? Chem. Geol. 209, 27-48.

Fritz, H., Abdel salam, M., Ali, K.A., Bingen, B., Collins, A.S., Fowler, A.R., Ghebreab,W., Hauzenberger, C. A., Johnson, P. R., Kusky, T. M., Macey, P., Muhongo, S.,Stern, R. J. and Viola, G., (2013): Orogen styles in the East African Orogen: a reviewof the Neoproterozoic to Cambrian tectonic evolution. J. Afr. Earth Sci. 86, 65-106.

Gill, R., (2010): Igneous rocks and processes: a practical guide. Wiley Blackwell, p. 460.

Gorton, M. P. and Schandal, E.S., (2000): From continents to island arcs: A geochemical index of tectonic setting for arc-related and within-plate felsic to intermediate volcanic rocks. The Canadian Mineralogist 38, 1065-1073.

Gradstein, F. M., Ogg, J.-G., Smith, A. G., Bleeker, W. and Lourens, L. J., (2004): A new geologic time scale with special reference to Precambrian and Neogene. Episodes 27, 83-100.

Green, T. H., (1980): Island arc and continent-building magmatism, a review ofpetrogenetic models based on experimental petrology and geochemistry. Tectonophysics 63, 367-385.

Grothaus, B., Eppler, D., Ehrlich, R., (1979): Depositional environments and structural implications of the Hammamat Formation, Egypt. Ann. Geol. Surv. Egypt 9, 564-590.

Grove, T. L., Elkins Tanton, L. T., Parman, S. W., Chatterjee, N., Müntener, G. and Gaetani, G.A., (2003): Fractional crystallization and mantle melting controls on calc-alkaline differentiation trends. Contrib. Miner. Petrol. 145, 515-533.

Hollings, P. and Wyman, D., (1999): Trace element and Sm-Nd systematics of volcanic and intrusive rocks from the 3 GaLumby Lake Greenstone belt, Superior Province: evidence for Archean plumearc interaction. Lithos 46, 189-213.

Irber, W., (1999): The lanthanide tetrad effect and its correlation with $\mathrm{K} / \mathrm{Rb}, \mathrm{Eu} / \mathrm{Eu}$, $\mathrm{Sr} / \mathrm{Eu}, \mathrm{Y} / \mathrm{Ho}$, and $\mathrm{Zr} / \mathrm{Hf}$ of evolving peraluminous granite suites. Geochim. Cosmochim. Acta 63, 489-508. 
Seddiek, et al.

Irvine, T. N. and Baragar, W. R. A., (1971): A guide to the chemical classification of the common volcanic rocks. Canadian Journal of Earth Sciences 8, 523-548.

Khalaf, E. A., (2012): Origin and evolution of post-collisional volcanism: an example from Neoproterozoic Dokhanvolcanics at Gabal Nugara area, Northeastern Desert, Egypt. Arab J. of Geosci. 5, 663-695.

Khalaf, E. A., (2013): Variations in eruptive style and depositional processes of Neopro-terozoic terrestrial volcano-sedimentary successions in the Hamid area, NorthEastern Desert, Egypt. J. Afr. Earth Sci. 83, 74-103.

Khalaf, E. A., Khalaf, M., and Oraby, F., (2011): Polybaric evolution of the volcanic rocks at Gabal Nuqara, North Eastern Desert, Egypt. In: Ray, J.et al. (Ed.), Topics in Igneous Petrology. Springer, Dordrecht/Heidelberg, 277-317.

Kröner, A., Todt, W., Hussein, I.M., Mansour, M., Rashwan, A., (1992): Dating of late Proterozoic ophiolites in Egypt and the Sudan using single zircon evaporation technique. Precamb. Res. 59, $15-32$.

Le Bas, M., Maitre, R. L., Streckeisen, A., Zanettin, B., IUGS Subcommission on the Systematics of Igneous Rocks, (1986): A chemical classification of volcanic rocks based on the total alkali-silica diagram. Journal of petrology. 27, 745-750.

Le Maitre, R. W., (1989): A Classification of Igneous Rocks and Glossary of Terms. Blackwell, Oxford, p. 193.

Liégeois, J. P. and Black, R., (1987): Alkaline magmatism subsequent to collision in the Pan- African belt of the Adrar des Iforas. In: Fitton, J.G., Upton, B.G.J. (Eds.), Alkaline Igneous Rocks. Geological Society of London, Special Publication 30, 381-401.

Liégeois, J. P., Navez, J., Black, R. and Hertogen, J., (1998): Contrasting origin of post-collision high$\mathrm{K}$ calc-alkaline and shoshonitic versus alkaline and peralkaline granitoids. The use of sliding normalization. Lithos 45, 1-28.

McPhie, J., Doyle, M. and Allen, R., (1993): Volcanic Textures: a Guide to the Interpretation of Textures in Volcanic Rocks. University of Tasmania Centre for Ore Deposit and Exploration Studies, Hobart, 198 p.

Miller, C. F., and Mittlefehldt, D. W., (1984): Extreme fractionation in felsic magma chambers: a product of liquid-state diffusion or fractional crystallization? Earth Planet Sci. Lett. 68, 151-158.

Moghazi, A. M., (1994): Geochemical and radiogenic isotope studies of some basement rocks at the Kid area, southeastern Sinai, Egypt. Unpublished Ph. D. Thesis, Alexandria Univ, Egypt, 377 pp.

Moghazi, A. M., (2003): Geochemistry and petrogenesis of a high-K calc- alkaline Dokhan Volcanic suite, South Safaga area, Egypt: the role of late Neoproterozoic crustal extension. Precamb. Res., 125, 161-178.

Mohammed, F. H., Moghazi, A. M. and Hassanen, M. A., (2000): Geochemistry, petrogenesis and tectonic setting of late Neoproterozoic Dokhan-type volcanic rocks in the Fatira area, eastern Egypt. Int. Journ. Earth Sciences 88, 764-777.

Nicolae, I., and Saccani, E., (2003): Petrology and geochemistry of the Late Jurassic calc-alkaline series associated to Middle Jurassic ophiolites in the South Apuseni Mountains (Romania). Schweiz. Mineral. Petrogr. Mitt. 83, 81-96.

Obeid, M. A. and Azer, M. K., (2015): Pan-African adakitic rocks of the north Arabian-Nubian Shield: petrological and geochemical constraints on the evolution of the Dokhan volcanics in the north Eastern Desert of Egypt. Int. J. Earth Sci., 104, 541-563.

Pearce, J. A., (1983): Role of the sub-continental lithosphere in magma genesis at active continental margins. In: Hawkesworth, C.J., Norry, M.J. (Eds.), Continental Basalts and Mantle Xenoliths. Shiva, Nantwich, 230-249.

Pearce, J. A., Harris, N. B. W. and Tindle, A. G. (1984): Trace element discrimination diagrams for the tectonic interpretation of granitic rocks. Journal of Petrology 25, 956-983.

Ressetar, R., Monard, J. R., (1983): Chemical composition and tectonic setting of the Dokhan Volcanic formation, Eastern Desert, Egypt. J. Afr. Earth Sci. 1, 103-112.

Rudnick R. L. and Fountain, D. M., (1995): Nature and composition of the continental crust: a lower crustal perspective. Reviews in Geophysics 33, 267-309.

Saunders, A. D., Tarney, J. and Weaver, D., (1980): Transverse geochemical variations across the Antarctic Peninsula: implications for the genesis of Cal-alkaline magmas. Earth Planet. Sci. Lett. $46,344-360$. 
Petrological and geochemical investigations on Dokhan Volcanics

Shearer, C. K., Papike, J. J., Laul, J. C., (1985): Chemistry of potassium feldspars from three zoned pegmatites, Black Hills, South Dakota: implications concerning pegmatite evolution. Geochim. Cosmochim. Acta 49, 663-673.

Stern, R. J., (1994): Arc assembly and continental collision in the Neoproterozoic East African Orogen: implications for the consolidation of Gondwanaland. Annual Reviews in Earth and Planetary Sciences 22, 319-351.

Stern, R. J., (2002): Crustal evolution in the east African Orogen: a neodymium isotope prospective. J. Afr. Earth Sci. 34, 109-117.

Stern, R. J., and Gottfried, D., (1986): Petrogenesis of late Precambrian (575-600 Ma) bimodal suite in northeast Africa. Contributions to Mineralogy and Petrology 92, 492-501.

Stern, R. J., Gottfried, D. and Hedge, C. E. (1984): Late Precambrian rifting and crustal evolution in the North Eastern Desert of Egypt. Geology 12, 168-172.

Stern, R. J., Hedge, C. E., (1985): Geochronologic and isotopic constraints on late Precambrian crustal evolution in the Eastern Desert of Egypt. Am. J. Sci. 285, 97-127.

Stern, R. J., (1981): Petrogenesis and tectonic setting of Late Precambrian ensimatic volcanic rocks, central Eastern Desert of Egypt. Precamb. Res. 16, 195-230.

Sun, S. S., and McDonough W.F., (1989): Chemical and isotopic systematics of oceanic basalts: implications for mantle composition and processes. In: Saunders A. D., and Norry, M. J., (eds.), Magmatism in the Ocean Basins: Geological Society of London Sp. Publ., 42, 313-345.

Taylor, S. R., (1965): The application of trace element data to problems in petrology. Phys. Chem.

Earth, 6, 133-213.

Watson, E. B., (1979): Zircon saturation in felsic liquids: experimental results and applications to trace element geochemistry. Contrib. Mineral Petrol 70, 407-419.

Wedepohl, K. H., (1995): The composition of the continental crust. Geochim. Cos-mochim. Acta 59, 1217-1232.

Wilde, S. A. and Youssef, K., (2000): Significance of SHRIMP U-Pb dating of the imperial porphyry and associated DokhanVolcanics, Gebel Dokhan, north Eastern Desert, Egypt. J. Afr. Earth Sci., 31(2), 403-413.

Wilson, M., (1989): Igneous petrogenesis - A global tectonic approach. Unwin Hyman Ltd., 466p. Winchester, J. A., Floyd, P.A., 1976. Geochemical magma type discrimination: application to altered and metamorphosed basic igneous rocks. Earth and Planet. Sci. Lett., 28, 459-469.

Wilson, M., (1994): Igneous Petrogenesis. Chapman and Hall, London, 466 p. 
Seddiek, et al.

دراسات بترولوجية وجيوكيميائية لبركانيات الاخان المتواجدة بمنطقة وادي أم سيدرا ووادي أم أسمر، شمال الصحراء الشرقية، مصر

صديق حمدي صديق 'و عادل حسن الافندي' و باهر عبد الحميد القليوبي`و حسن علي عليوة'و حسام انور خميس'

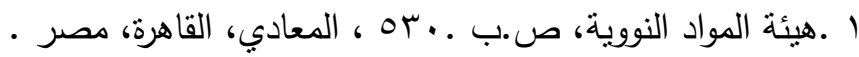

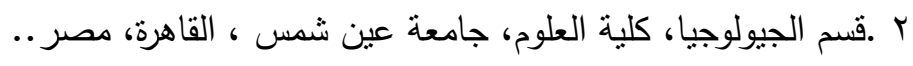

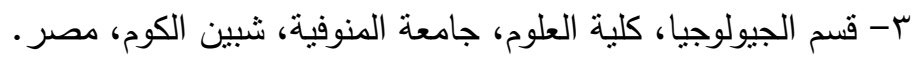

\section{الخلاصة}

يتتاول هذا البحث دراسة جيولوجية وبتروجرافية وجيوكيميائية لبركانيات الدخان في منطقة وادي أم سيدرا ووادي أم اسمر

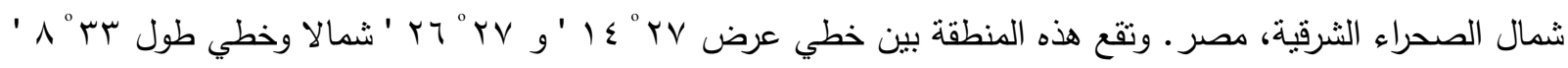

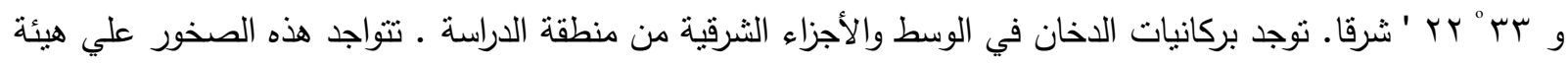

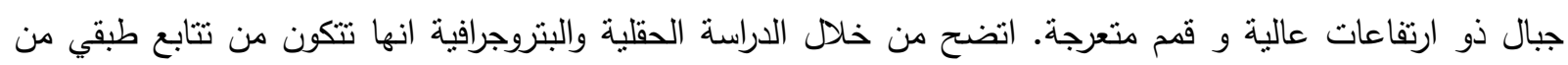

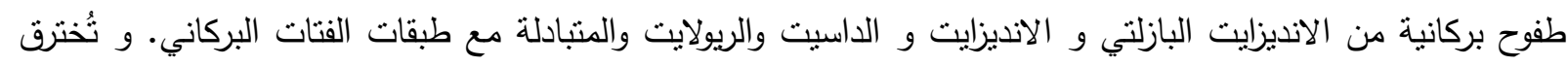
هذه الصخور بالصخور الجرانيتيه المجاورة لها المتنثلة في المونزوجرانيت والسيانوجرانيت وجرانيت الفلسبار القلوي وأيضا

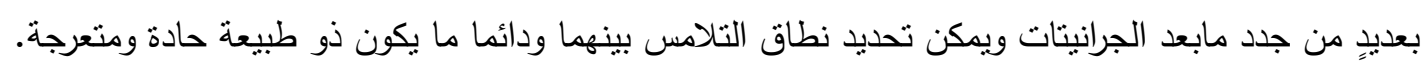

وقد أظهرت الدراسة الجيوكيميائية للعناصر الرئيسية والنادرة لحمم بركانيات الدخان انها قد نشأت من صهير كلسى

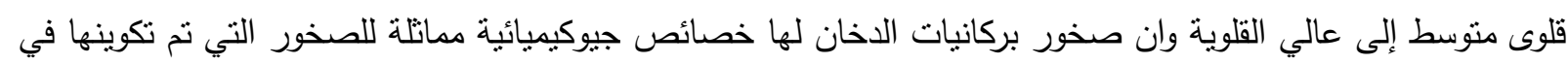

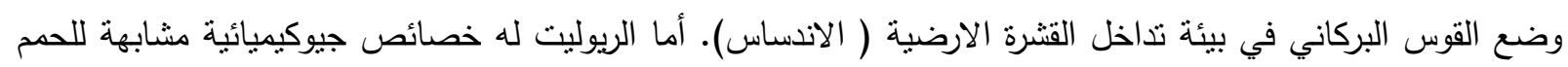

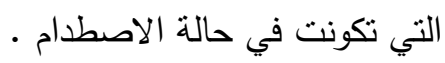

يمكن تفسير التغير المنهجي للعناصر الرئيسية والنادرة لعينات بركانيات الدخان التي تم درستها من خلال مفهوم التبلور

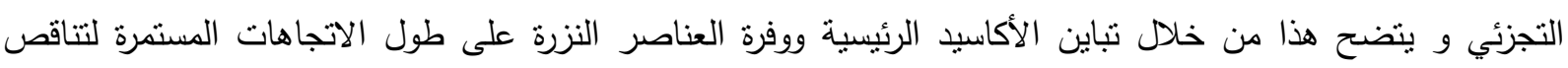

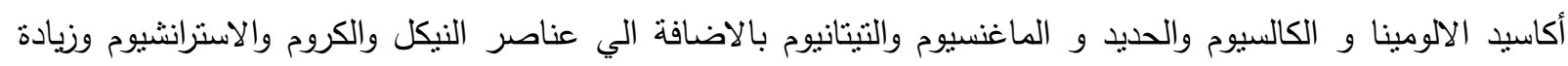

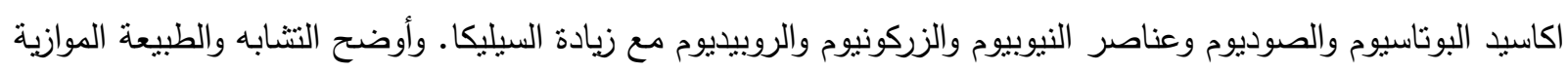

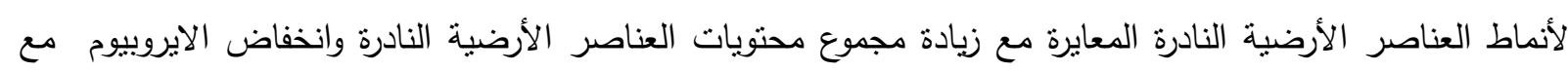

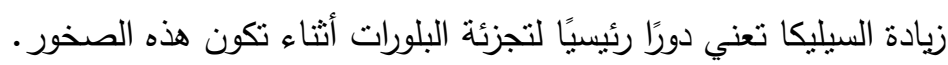

